\title{
ON THE STRUCTURE OF ALMOST EINSTEIN MANIFOLDS
}

\author{
GANG TIAN AND BING WANG
}

Contents

1. Introduction

2. Elementary estimates

3. A pseudo-locality theorem

4. Curvature, distance and volume estimates

5. Structure of limit space

5.1. Riemannian case

5.2. Kähler case

6. Examples

6.1. Smooth minimal varieties of general type

6.2. Fano manifolds

Acknowledgment

References

\section{INTRODUCTION}

The regularity theory for non-collapsed Einstein manifolds has attracted many studies in past two decades, e.g., [2], [3, 37, 6], 8], etc. This theory and its extensions have played a crucial role in Kähler geometry, e.g., in constructing canonical metrics on Fano surfaces (cf. 37, [16]).

Motivated by the study in Kähler geometry, in this paper, we prove new regularity results on the Gromov-Hausdorff limits of Riemannian manifolds with Ricci curvature bounded from below and which are weakly Einstein in an appropriate sense.

To be precise, we assume that $\left(X_{i}, x_{i}, g_{i}\right)$ is a sequence of non-collapsed Riemannian manifolds of dimension $m$ such that Ric $\geq-(m-1) \lambda$ for some uniformly bounded constant $\lambda$. The well-known Gromov compactness theorem states that by taking a subsequence if necessary, $\left(X_{i}, x_{i}, g_{i}\right)$ converges to a length space $(\bar{X}, \bar{x}, \bar{g})$ in the Gromov-Hausdorff topology. A basic problem in the metric geometry concerns the regularity of the limit $(\bar{X}, \bar{x}, \bar{g})$. Note that $(\bar{X}, \bar{x}, \bar{g})$ is merely a length space in the Gromov compactness theorem. The fundamental work of Cheeger-Colding [6] shows initial and crucial structure properties for $(\bar{X}, \bar{x}, \bar{g})$. In particular, it follows from [6] that tangent cones exist at every point $y \in \bar{X}$. Using these tangent cones,

Received by the editors February 24, 2013 and, in revised form, September 29, 2014.

2010 Mathematics Subject Classification. Primary 53Cxx; Secondary 35Jxx.

The first author was partially supported by NSF Grants DMS-0804095, DMS-1309359 and an NSFC Grant.

The second author was partially supported by NSF Grant DMS-1006518 and funds from SCGP. 
they gave a regular-singular decomposition of $\bar{X}$. A point $y \in \bar{X}$ is called regular or belongs to the regular part $\mathcal{R}$ if every tangent cone at $y$ is isometric to the Euclidean space $\left(\mathbb{R}^{m}, 0, g_{\mathbb{E}}\right)$. A point $y \in X$ is called singular or belongs to the singular part $\mathcal{S}$ if it is not regular, i.e., at $y$, there exists some tangent cone $(\hat{Y}, \hat{y}, \hat{g})$ which is not isometric to the Euclidean space. Clearly, we have $\bar{X}=\mathcal{R} \cup \mathcal{S}$. In general, it is unknown if $\mathcal{R}$ is open and even if it is open, $(\mathcal{R}, \bar{g})$ may not be a smooth Riemannian manifold. If $g_{i}$ has uniformly bounded Ricci curvature, then Cheeger-Colding proved that $\mathcal{R}$ is an open manifold and $\mathcal{S}$ has Hausdorff codimension at least 2 . Moreover, $\bar{g}$ is a $C^{1, \alpha}$-smooth metric. Furthermore, if $\left(X_{i}, g_{i}\right)$ is an Einstein manifold, then the convergence to $\bar{X}$ restricted to $\mathcal{R}$ is actually in the $C^{\infty}$-topology and $\bar{g}$ is a smooth Einstein metric in $\mathcal{R}$ because of the regularity results from the elliptic partial differential equation (PDE) theory. However, in general, even if the convergence is weak, $\mathcal{R}$ can still possibly be a smooth manifold. In this paper, we study when the limit can have smooth $\mathcal{R}$ and $\bar{g}$ is an Einstein metric even if the convergence $\left(X_{i}, x_{i}, g_{i}\right) \rightarrow(\bar{X}, \bar{x}, \bar{g})$ is only in the weak topology, say the GromovHausdorff topology. Our study is analogous to the standard regularity problem in studying weak solutions for elliptic PDEs. In the case of the Einstein equation, because of its invariance under diffeomorphisms, there does not exist a good notion of weak solutions. Therefore, we first need to make clear what we mean by Einstein metrics in the weak sense. Now let us introduce the notion of almost all Einstein manifolds we want to study.

Definition 1. A sequence of closed Riemannian manifolds $\left(X_{i}^{m}, x_{i}, g_{i}\right)$ is called almost Einstein if the following conditions are satisfied:

- $\operatorname{Ric}\left(g_{i}\right)+g_{i} \geq 0$.

- $x_{i} \in X_{i}$, and $\left|B_{g_{i}}\left(x_{i}, 1\right)\right|_{d \mu_{g_{i}}} \geq \kappa$.

- The flow $\frac{\partial}{\partial t} g=-$ Ric $+\lambda_{i} g$ has a solution $g(t)$ with $g(0)=g_{i}$ on $X_{i} \times[0,1]$, where $\lambda_{i} \in[-1,1]$ is a constant. Moreover, $E_{i}=\int_{0}^{1} \int_{X_{i}}\left|R-m \lambda_{i}\right| d \mu d t \rightarrow 0$.

Note that the non-collapsed condition is included in our definition. This is because the condition $\int_{0}^{1} \int_{X_{i}}\left|R-m \lambda_{i}\right| d \mu_{g_{i}} d t \rightarrow 0$ is not sufficient for proving the following results if collapsing occurs. However, we will not discuss this further in the current paper.

Clearly, if $\int_{0}^{1} \int_{X_{i}}\left|R-m \lambda_{i}\right| d \mu_{g_{i}} d t \equiv 0$, then this sequence is exactly a sequence of non-collapsed Einstein manifolds with bounded Einstein constants. Such a sequence was extensively studied in the literature. In fact, the condition $\int_{0}^{1} \int_{X_{i}} \mid R-$ $m \lambda_{i} \mid d \mu_{g_{i}} d t \rightarrow 0$ is crucial in establishing the regularity of $\mathcal{R}$. It turns out that almost Einstein limits have most known properties of Einstein limits. Our first theorem is as follows.

Theorem 1 (Structure theorem in Riemannian case). Suppose $\left(X_{i}^{m}, x_{i}, g_{i}\right)$ is a sequence of almost Einstein manifolds. Let $(\bar{X}, \bar{x}, \bar{g})$ be a Gromov-Hausdorff limit of $\left(X_{i}, x_{i}, g_{i}\right)$, and $\bar{\lambda}$ be the limit of $\lambda_{i}$.

Then the limit space $(\bar{X}, \bar{x}, \bar{g})$ is a metric space with disjoint decomposition $\bar{X}=$ $\mathcal{R} \cup \mathcal{S}$, where $\mathcal{R}$ is the regular part of $\bar{X}$, and $\mathcal{S}$ is the singular part of $\bar{X}$. They 
satisfy the following properties:

- $(\mathcal{R}, \bar{g})$ is a smooth, convex, open Riemannian manifold.

- $\operatorname{Ric}(\bar{g})+\bar{\lambda} \bar{g}=0$.

- If $0<p<1$ and $\rho \geq 1$, then $\int_{\mathcal{R} \cap B(\bar{x}, \rho)}|R m|^{p} d \mu<C(m, \kappa, p, \rho)$.

- If $y \in \mathcal{S},(\hat{X}, \hat{y}, \hat{g})$ is a tangent space of $\bar{X}$ at the point $y$, then

$$
d_{G H}\left(\left(B_{\hat{g}}(\hat{y}, 1), \hat{g}\right),\left(B(0,1), g_{\mathbb{E}}\right)\right)>\bar{\epsilon}(m),
$$

where $B(0,1)$ is the standard unit ball in $\mathbb{R}^{m}$.

- $\operatorname{dim}_{\mathcal{H}} \mathcal{S} \leq m-2$.

Note that the convexity of $\mathcal{R}$ and the integral bound of $|R m|$ follow directly from the work of [20] and [12, respectively. We list these results here just for completeness of the known results of the Einstein limit.

We observe that if $\left(M_{i}, x_{i}, g_{i}\right)$ is a sequence of Kähler manifolds 1 then by a result of the first author and Zhang (cf. [40]), the Ricci flow $\frac{\partial}{\partial t} g=-R i c+\lambda_{i} g$ has a solution with $g(0)=g_{i}$ on $M_{i} \times[0,1]$ so long as $\lambda_{i}\left[\omega_{i}\right]+\left(e^{\lambda_{i} t}-1\right) c_{1}\left(M_{i}\right)>0$, where $\omega_{i}$ denotes the Kähler form of $g_{i}$. Moreover, if $R-n \lambda_{i} \geq 0$ and its average tends to zero as $i$ goes to infinity, then one can show that $E_{i}$ tends to zero. Thus, the third condition of Definition 1 is essentially automatic if $R-n \lambda_{i} \geq 0$ and its average tends to zero. This shows that the Kähler case is better behaved. A natural question is whether the same holds for general Riemannian metrics with Ricci curvature bounded from below. More precisely, can one solve the above Ricci flow with initial value $g_{0}$ in $[0, a]$ such that $a$ depends only on the lower bound of the Ricci curvature of $g_{0}$ ?

The following theorem strengthens Theorem 1 for Kähler manifolds. We say that a sequence of closed Kähler manifolds $\left(M_{i}^{n}, x_{i}, g_{i}, J_{i}\right)$ is almost Kähler-Einstein if it is almost Einstein of dimension $m=2 n$ and satisfies $F_{i}=\int_{M_{i}}\left|R i c-\lambda_{i} g_{i}\right| d \mu_{g_{i}} \rightarrow 0$.

Theorem 2 (Structure theorem in Kähler case). Suppose $\left(M_{i}^{n}, x_{i}, g_{i}, J_{i}\right)$ is a sequence of almost Kähler Einstein manifolds. Let $(\bar{M}, \bar{x}, \bar{g})$ be a Gromov-Hausdorff limit of $\left(M_{i}, x_{i}, g_{i}\right)$, and $\bar{\lambda}$ be the limit of $\lambda_{i}$.

Then the limit space $(\bar{M}, \bar{x}, \bar{g})$ is a metric space with the regular-singular disjoint decomposition $\bar{M}=\mathcal{R} \cup \mathcal{S}$. They satisfy the following properties:

- There exists a complex structure $\bar{J}$ on $\mathcal{R}$ such that $(\mathcal{R}, \bar{g}, \bar{J})$ is a smooth, convex, open Kähler manifold.

- $\operatorname{Ric}(\bar{g})+\bar{\lambda} \bar{g}=0$.

- If $0<p<2$ and $\rho \geq 1$, then $\int_{\mathcal{R} \cap B(\bar{x}, \rho)}|R m|^{p} d \mu<C(n, \kappa, p, \rho)$.

- If $y \in \mathcal{S},(\hat{M}, \hat{y}, \hat{g})$ is a tangent space of $\bar{M}$ at the point $y$, and then

$$
d_{G H}\left(\left(B_{\hat{g}}(\hat{y}, 1), \hat{g}\right),\left(B(0,1), g_{\mathbb{E}}\right)\right)>\bar{\epsilon}(2 n),
$$

where $B(0,1)$ is the standard unit ball in $\mathbb{R}^{2 n}$.

- $\operatorname{dim}_{\mathcal{H}} \mathcal{S} \leq 2 n-4$.

\footnotetext{
${ }^{1}$ In this paper, we always use $X$ to denote a Riemannian manifold of dimension $m$, and use $M$ to denote a Kähler manifold of complex dimension $n$ and real dimension $m=2 n$.
} 
Our proof of the above theorems is based on the celebrated works of [6], [8, [30], et al. We need to establish two new technical results. The first one is a pseudolocality property (Theorem 3.1) which is similar to Theorems 10.1 and 10.3 of 30. This property basically says that the curvature can be bounded locally along the Ricci flow whenever the initial metric has a Ricci lower bound and almost-Euclidean volume ratios locally. The structure of our proof for this pseudo-locality property resembles the one for Theorem 10.1 in [30]. However, we need new ingredients in our proof since we do not assume the sharp isoperimetric constant as Perelman has in 30. One of the key points in Perelman's proof is to show the continuity of Perelman's functional by using the assumption on the sharp isoperimetric constant, while we have found a very different way to do it without using the isoperimetric constant. The fundamental results in [19, 6], and [7, particularly, the volume continuity theorem of Colding [19, play essential roles for our proof of the functional continuity. The second technical difficulty is a delicate bound of the GromovHausdorff distance between metrics along the Ricci flow (cf. Theorem 4.2). This bound plays a role similar to that of the gap theorem for Einstein limits and is crucial for us to finish the proof of Theorems 1 and 2 .

The motivation for developing Theorems 1 and 2 comes from the study of Kähler geometry. There are Kähler examples where the general compactness theorems of Kähler Einstein metrics fail to work and our Theorems work well. Therefore, our compactness theorem is a necessary step for further understanding of Kähler geometry. Two examples will be discussed in details at the end of this paper (see Section 6). One concerns the uniformization of manifolds of general type, and the other concerns the existence of Kähler-Einstein metrics Fano manifolds with K-energy bounded from below or some condition of this sort.

The organization of this paper is as follows. In Section 2, we discuss some standard estimates which will be repeatedly used in the whole paper. In Section 3, we prove a new pseudo-locality result, i.e., Theorem 3.1 Using this new pseudolocality, the pseudo-locality theorem, the gap theorem, and the fact that scalar curvature is almost constant to show the structure theorems in both Riemannian and Kähler cases. Finally, in Section 6, we construct examples of almost Kähler Einstein manifolds and discuss the applications of our structure theorems to Kähler geometry.

\section{Elementary estimates}

Before we discuss the details, let us fix some notations. We assume $X$ to be a closed Riemannian manifold of dimension $m \geq 3, M$ to be a closed Kähler manifold of complex dimension $n \geq 2$, and real dimension $m=2 n \geq 4$. We denote the volume of the standard unit ball in $\mathbb{R}^{m}$ by $\omega_{m}$. We say $A<<B$ for two positive quantities $A$ and $B$ if there is a universal small constant $c=c(m)$ such that $A<c B$. If not mentioned in particular, the constant $C$ may be different from line to line.

In this paper, we often assume $\{(X, g(t)), 0 \leq t \leq 1\}$ satisfies the evolution equation

$$
\frac{\partial}{\partial t} g=-R i c+\lambda_{0} g
$$

for some constant $\lambda_{0}$ with $\left|\lambda_{0}\right| \leq 1$. Note that this flow may not preserve the volume. However, by abuse of notation, we also call (1) a normalized Ricci flow 
solution. Define

$$
\tilde{g}(s) \triangleq\left\{\begin{array}{l}
\left(1-2 \lambda_{0} s\right) g\left(\frac{\log \left(1-2 \lambda_{0} s\right)}{-\lambda_{0}}\right), \text { if } \lambda_{0} \neq 0 \\
g(2 s), \text { if } \lambda_{0}=0 .
\end{array}\right.
$$

Then $\frac{\partial}{\partial s} \tilde{g}=-2 \operatorname{Ric}(\tilde{g})$, which is the (unnormalized) Ricci flow equation. Clearly, $\tilde{g}(0)=g(0)$. For simplicity of notation, define $h_{i j} \triangleq R_{i j}-\lambda_{0} g_{i j}, H \triangleq R-m \lambda_{0}$. Simple calculation yields

$$
\frac{\partial}{\partial t} h_{i j}=\frac{1}{2} \Delta h_{i j}+R_{p i j q} h_{i j}-h_{i p} h_{p j}
$$

which implies

$$
\frac{\partial}{\partial t}|h| \leq \frac{1}{2} \Delta|h|+|R m||h|
$$

Taking the trace of (3), we obtain

$$
\frac{\partial}{\partial t} H=\frac{1}{2} \Delta H+|h|^{2}+\lambda_{0} H
$$

Define $H_{\min }(t) \triangleq \min _{x \in X} H(x, t)$. Applying the maximum principle to (5), we obtain

$$
\frac{\partial}{\partial t} H_{\text {min }}(t) \geq \lambda_{0} H_{\text {min }}(t) \Rightarrow H_{\text {min }}(t) \geq e^{\lambda_{0} t} H_{\min }(0) .
$$

In particular, the condition $H \geq 0$ is preserved by the normalized Ricci flow (1).

It follows from (11) that the distance derivative with respect to time is controlled by the $\left|R i c-\lambda_{0} g\right|$ along the shortest geodesic. However, a more delicate analysis shows that the lower bound of the distance derivative depends only on the local Ricci upper bound around the end points.

Proposition 2.1 (cf. Section 17 of [27, or Lemma 8.3(b) of [30]). Suppose $\{(X, g(t)), 0 \leq t \leq 1\}$ is a normalized Ricci flow solution $\frac{\partial}{\partial t} g=-$ Ric $+\lambda_{0} g$ with $\left|\lambda_{0}\right| \leq 1$. Suppose $0 \leq t_{0} \leq 1, x_{1}, x_{2}$ are two points in $X$ such that $\operatorname{Ric}\left(x, t_{0}\right) \leq$ $(m-1) K$ when $d_{g\left(t_{0}\right)}\left(x, x_{1}\right)<r_{0}$ or $d_{g\left(t_{0}\right)}\left(x, x_{2}\right)<r_{0}$. Then

$$
\left.\frac{d}{d t} d_{g(t)}\left(x_{1}, x_{2}\right)\right|_{t=t_{0}} \geq \frac{1}{2} \lambda_{0} d_{g\left(t_{0}\right)}\left(x_{1}, x_{2}\right)-(m-1)\left(\frac{2}{3} K r_{0}+r_{0}^{-1}\right) .
$$

Proof. Without loss of generality, one can assume $t_{0}=0$. Then the proof is just an application of the renormalization equation (2) and Lemma 8.3(b) of [30].

Suppose $\Omega$ is a compact manifold with a boundary. The following lemmas are standard (cf. [28]).

Lemma 2.1. Suppose $(X, g)$ is a complete manifold, $x_{0} \in X, 0<r \leq 1$. Suppose $r^{-m}\left|B\left(x_{0}, r\right)\right| \geq \kappa$ and $r^{2}$ Ric $\geq-(m-1)$ in $B\left(x_{0}, 2 r\right)$. Let $\Omega=B\left(x_{0}, r\right)$. Then the following properties are satisfied:

- The isoperimetric constant of $\Omega$ is uniformly bounded by $C_{I}=C_{I}(m, \kappa)$.

- The Sobolev constant of $\Omega$ is uniformly bounded by $C_{S}=C_{S}(m, \kappa)$.

- The Neuman Poincaré constant of $\Omega$ is uniformly bounded by $C_{P}=C_{P}(m, \kappa)$. 
Lemma 2.2. Suppose $(X, g)$ is a complete Riemannian manifold, $x_{0} \in X$. Suppose the following conditions are satisfied:

- For every $0<r<2$, we have $C_{V}^{-1}<\left|B\left(x_{0}, r\right)\right| r^{-m}<C_{V}$.

- The Sobolev constant of $B\left(x_{0}, 2\right)$ is bounded by $C_{S}$.

- The Poincaré constant of $B\left(x_{0}, 2\right)$ is bounded by $C_{P}$.

- $|a|+|b|<C_{F}$ on $B\left(x_{0}, 2\right)$ where $a, b$ are two constants.

Suppose $\varphi \geq 0$ satisfies the inequality $(-\Delta+a) \varphi \geq b$ in the distribution sense; then

$$
\int_{B\left(x_{0}, 1\right)} \varphi \leq C\left(1+\inf _{B\left(x_{0}, \frac{1}{2}\right)} \varphi\right),
$$

where $C=C\left(m, C_{V}, C_{S}, C_{P}, C_{F}\right)$. Consequently, for every $0<\rho<1$, we have

$$
\rho^{-m} \int_{B\left(x_{0}, \rho\right)} \varphi \leq C\left(\rho^{2}+\inf _{B\left(x_{0}, \frac{\rho}{2}\right)} \varphi\right),
$$

where $C$ is the same constant as in (8).

Proof. Let $\bar{\varphi}=\varphi+C_{F}$. We compute

$$
\begin{aligned}
(-\Delta+a) \bar{\varphi} & \geq b+a C_{F}=C_{F}\left(a+C_{F}^{-1} b\right) \geq-C_{F}\left|a+C_{F}^{-1} b\right| \geq-\left|a+C_{F}^{-1} b\right| \bar{\varphi} \\
& \geq-(|a|+1) \bar{\varphi} .
\end{aligned}
$$

It follows

$$
\Delta \bar{\varphi} \leq(2|a|+1) \bar{\varphi} \leq\left(2 C_{F}+1\right) \bar{\varphi} .
$$

By the standard De Giorgi-Nash-Moser iteration (cf. Lemma 11.2 of [28]), we have

$$
\int_{B\left(x_{0}, 1\right)} \bar{\varphi} \leq C \inf _{B\left(x_{0}, \frac{1}{2}\right)} \bar{\varphi}
$$

for some $C$ depending on $m, C_{V}, C_{S}, C_{P}$, and $C_{F}$. This in turn implies (8).

Fix $0<\rho<1$. Let $\tilde{g}=\rho^{-2} g$. By the scaling property of the Laplacian operator, we see that

$$
(-\Delta+a) \varphi \geq b \Leftrightarrow-\rho^{-2} \Delta_{\tilde{g}} \varphi+a \varphi \geq b \Leftrightarrow-\Delta_{\tilde{g}}\left(\rho^{-2} \varphi\right)+\rho^{2} a\left(\rho^{-2} \varphi\right) \geq b .
$$

Let $\tilde{\varphi}=\rho^{-2} \varphi$, and we have

$$
-\Delta_{\tilde{g}} \tilde{\varphi}+\rho^{2} a \tilde{\varphi} \geq b .
$$

Consider this system under the metric $\tilde{g}$. The four estimates hold for this new system, so we obtain

$$
\int_{B_{\tilde{g}}\left(x_{0}, 1\right)} \tilde{\varphi} \leq C\left(1+\inf _{B_{\tilde{g}}\left(x_{0}, \frac{1}{2}\right)} \tilde{\varphi}\right),
$$

which is the same as (9) since $\tilde{\varphi}=\rho^{-2} \varphi$.

Combing Lemmas 2.1 and 2.2 we obtain the following Proposition, which is very useful in the study of a boundary estimate. 
Proposition 2.2. Suppose $\left(X, x_{0}, g\right)$ is a complete Riemannian manifold. Suppose $0<r \leq 1, r^{-m}\left|B\left(x_{0}, r\right)\right| \geq \kappa, r^{2}$ Ric $\geq-(m-1)$ on $B\left(x_{0}, 2 r\right)$. Suppose $\varphi \geq 0$ satisfies the inequality $(-\Delta+a) \varphi \geq b$ for $|a|+|b|<C_{F}$. Then for every $0<\rho \leq r$, we have

$$
\rho^{-m} \int_{B\left(x_{0}, \rho\right)} \varphi \leq C\left(\rho^{2}+\inf _{B\left(x_{0}, \frac{\rho}{2}\right)} \varphi\right),
$$

for some constant $C=C\left(m, \kappa, C_{F}\right)$.

\section{A PSEUdo-LOCALity THEOREM}

Under the Ricci flow, an "almost-Euclidean" region cannot become singular suddenly. This is the principle of pseudo-locality as stated by Perelman in Section 10 of [30]. Perelman developed some pseudo-locality theorems by regarding "almost" as close of isoperimetric constant and scalar lower bound. Of course, this is not the unique "almost-Euclidean" condition. In this section, we will develop similar pseudo-locality properties by explaining "almost-Euclidean" balls as balls whose volume ratio and Ricci lower bound is close to that of the Euclidean balls.

Proposition 3.1 (A pseudo-locality property; compare Theorems 10.1 and 10.3 of Perelman [30]). For every $0<\alpha<\frac{1}{100 m}$, there exist constants $\delta=\delta(m, \alpha), \epsilon=$ $\epsilon(m, \alpha)$ with the following properties:

Suppose $\{(X, g(t)), 0 \leq t \leq 1\}$ is a Ricci flow solution, $x_{0} \in X$. Suppose

$$
\begin{aligned}
& \operatorname{Ric}(x, 0) \geq-(m-1) \delta^{4}, \quad \forall x \in B_{g(0)}\left(x_{0}, \delta^{-1}\right) . \\
& \delta^{m}\left|B_{g(0)}\left(x_{0}, \delta^{-1}\right)\right|_{d \mu_{g(0)}} \geq(1-\delta) \omega_{m} .
\end{aligned}
$$

Then we have

$$
\begin{aligned}
& \left|B_{g(t)}(x, \sqrt{t})\right|_{d \mu_{g(t)}} \geq \kappa^{\prime} t^{\frac{m}{2}} \\
& |R m|(x, t) \leq \alpha t^{-1}+\epsilon^{-2}, \quad \forall x \in B_{g(t)}\left(x_{0}, \sqrt{t}\right), t \in\left(0, \epsilon^{2}\right],
\end{aligned}
$$

where $\kappa^{\prime}=\kappa^{\prime}(m)$ is a universal constant.

Proof. We only prove (14). The proof of (13) follows verbatim.

If the statement was false, we can find a sequence of $\delta_{k}, \epsilon_{k} \rightarrow 0, x_{k} \in X_{k}$ such that (11) and (12) hold. However, (14) are violated.

Following the proof of Perelman's pseudo-locality theorem, we can find a sequence of functions $u_{k}$ which are compactly supported on $B\left(x_{k}, 1\right)$ and satisfy (see the end of the proof of Theorem 10.1 of [30])

$$
\begin{aligned}
& \int_{B\left(x_{k}, 1\right)} u_{k}=1, \\
& \int_{B\left(x_{k}, 1\right)}\left\{\frac{1}{2}\left|\nabla f_{k}\right|^{2}+f_{k}-m\right\} u_{k} \leq-\eta<0,
\end{aligned}
$$


where $u_{k}=(2 \pi)^{-\frac{m}{2}} e^{-f_{k}}$. Of course, here we regard $d \mu_{g_{k}(0)}$ as the default measure. Let $\bar{u}_{k}=\sqrt{u_{k}}$. These equations can be written as

$$
\begin{aligned}
& \int_{B\left(x_{k}, 1\right)} \bar{u}_{k}^{2}=1, \\
& \int_{B\left(x_{k}, 1\right)}\left\{2\left|\nabla \bar{u}_{k}\right|^{2}-2 \bar{u}_{k}^{2} \log \bar{u}_{k}-m(1+\log \sqrt{2 \pi}) \bar{u}_{k}^{2}\right\} \leq-\eta .
\end{aligned}
$$

Denote by $\mathcal{F}_{k}\left(u_{k}\right)$ the integral

$$
\int_{B\left(x_{k}, 1\right)}\left\{2\left|\nabla \bar{u}_{k}\right|^{2}-2 \bar{u}_{k}^{2} \log \bar{u}_{k}-m(1+\log \sqrt{2 \pi}) \bar{u}_{k}^{2}\right\} .
$$

Clearly, $\mathcal{F}_{k}$ is a functional on the space of functions $\bar{u} \in W_{0}^{1,2}\left(B\left(x_{k}, 1\right)\right)$ satisfying $\int_{B\left(x_{k}, 1\right)} \bar{u}^{2}=1$. By the result of Rothaus 33, we see that $\mathcal{F}_{k}$ has a minimizer $\varphi_{k}$, which satisfies the Euler-Lagrange equation in $B\left(x_{k}, 1\right)$,

$$
-2 \Delta \varphi_{k}-2 \varphi_{k} \log \varphi_{k}-m(1+\log \sqrt{2 \pi}) \varphi_{k}=\lambda_{k} \varphi_{k}
$$

Note that $\varphi_{k}$ is continuous on the closure of $B\left(x_{k}, 1\right)$ and vanishes on the boundary of $B\left(x_{k}, 1\right)$, by the second lemma on page 113 of [33]. Therefore, by trivial extension, we can regard $\varphi_{k}$ as a function defined on $B\left(x_{k}, 2\right)$. On one hand, by the choice of $\lambda_{k}$, we have

$$
\lambda_{k}=\mathcal{F}\left(\varphi_{k}\right) \leq \mathcal{F}\left(\bar{u}_{k}\right) \leq-\eta<0 .
$$

On the other hand, integrating (17) over $B\left(x_{k}, 1\right)$ implies

$$
\begin{aligned}
\lambda_{k} & +m(1+\log \sqrt{2 \pi}) \\
& =\int 2\left|\nabla \varphi_{k}\right|^{2}-2 \int \varphi_{k}^{2} \log \varphi_{k} \\
& \geq \int 2\left|\nabla \varphi_{k}\right|^{2}-2 \int \varphi_{k}^{2} \cdot\left(\frac{m}{2 e} \varphi_{k}^{\frac{2}{m}}\right) \\
& =\int 2\left|\nabla \varphi_{k}\right|^{2}-\frac{m}{e} \int \varphi_{k} \cdot \varphi_{k}^{\frac{m+2}{m}}
\end{aligned}
$$

In the third step, we used the fact $\log x \leq \frac{m}{2 e} x^{\frac{2}{m}}$ for every positive $x$. Plugging the Hölder inequality into (18) yields

$$
\begin{aligned}
\lambda_{k} & +m(1+\log \sqrt{2 \pi}) \\
& \geq \int 2\left|\nabla \varphi_{k}\right|^{2}-\frac{m}{e}\left(\int \varphi_{k}^{\frac{2 m}{m-2}}\right)^{\frac{m-2}{2 m}} \cdot\left(\int \varphi_{k}^{2}\right)^{\frac{m+2}{2 m}} \\
& =\int 2\left|\nabla \varphi_{k}\right|^{2}-2 \cdot \frac{m}{2 e}\left(\int \varphi_{k}^{\frac{2 m}{m-2}}\right)^{\frac{m-2}{2 m}} \\
& \geq \int 2\left|\nabla \varphi_{k}\right|^{2}-\left\{a^{2}\left(\int \varphi_{k}^{\frac{2 m}{m-2}}\right)^{\frac{m-2}{m}}+\frac{m^{2}}{4 a^{2} e^{2}}\right\},
\end{aligned}
$$


where $a$ is a positive constant to be determined. Applying Lemma 2.1. we obtain a uniform bound for the Sobolev constant of $B\left(x_{k}, 1\right)$. It follows that

$$
\left(\int_{B\left(x_{k}, 1\right)} \varphi_{k}^{\frac{2 m}{m-2}}\right)^{\frac{m-2}{m}} \leq C_{S} \int_{B\left(x_{k}, 1\right)}\left(\varphi_{k}^{2}+\left|\nabla \varphi_{k}\right|^{2}\right) .
$$

Let $a^{2}=\frac{2}{C_{S}}$ and put (20) into (19); then we obtain

$$
\begin{aligned}
\lambda_{k}+m(1+\log \sqrt{2 \pi}) & \geq\left(2-a^{2} C_{S}\right) \int\left|\nabla \varphi_{k}\right|^{2}-\left(a^{2} C_{S}+\frac{m^{2}}{4 a^{2} e^{2}}\right) \\
& =-\left(2+\frac{m^{2} C_{S}}{8 e^{2}}\right) .
\end{aligned}
$$

Recalling that $\lambda_{k} \leq-\eta<0$, from (21) we see that there exists a constant $C_{\lambda}$, which depends on $m, C_{S}$, such that

$$
\left|\lambda_{k}\right|<C_{\lambda}
$$

Note that the Euler-Lagrange equation of $\varphi_{k}$ can be written as

$$
-\Delta \varphi_{k}=\left(\frac{1}{2}\left(m+m \log \sqrt{2 \pi}+\lambda_{k}\right)+\log \varphi_{k}\right) \varphi_{k} .
$$

Define $\bar{\varphi}_{k} \triangleq \max \left\{\varphi_{k}, 1\right\}$. Since $\log x \leq \frac{m}{2 e} x^{\frac{2}{m}}$ for every $x>0$, it follows from (23) that $\bar{\varphi}_{k}$ satisfies the inequality

$$
-\Delta \bar{\varphi}_{k} \leq \frac{1}{2}\left(m+m \log \sqrt{2 \pi}+\lambda_{k}+\frac{m}{e} \bar{\varphi}_{k}^{\frac{2}{m}}\right) \bar{\varphi}_{k}
$$

in the distribution sense. Clearly, we can uniformly bound the $L^{m}\left(B\left(x_{k}, 2\right)\right)$-norm of

$$
\frac{1}{2}\left(m+m \log \sqrt{2 \pi}+\lambda_{k}+\frac{m}{e} \bar{\varphi}_{k}^{\frac{2}{m}}\right),
$$

where $m>\frac{m}{2}$. Note that $B\left(x_{k}, 2\right)$ has a uniform Sobolev constant $C_{S}$. Then the standard Moser iteration implies that

$$
\left\|\bar{\varphi}_{k}\right\|_{C^{0}\left(B\left(x_{k}, 1\right)\right)} \leq C\left\|\bar{\varphi}_{k}\right\|_{L^{2}\left(B\left(x_{k}, 2\right)\right)} \leq C
$$

which in turn implies

$$
\left\|\varphi_{k}\right\|_{C^{0}\left(B\left(x_{k}, 1\right)\right)} \leq C_{1}=C_{1}\left(m, C_{S}, C_{\lambda}\right) .
$$

Recalling that the Ricci curvature is uniformly bounded from below on $B\left(x_{k}, 2\right)$, the estimate of Cheng-Yau (cf. [17, Section 6 of [28]) implies that

$$
\left|\nabla \varphi_{k}(x)\right| \leq C_{2}\left(m, d\left(x, \partial B\left(x_{k}, 1\right)\right)\right), \quad \forall x \in B\left(x_{k}, 1\right) .
$$

In view of the non-collapsed condition and the Ricci lower bound, we have the convergence in the pointed Gromov-Hausdorff topology,

$$
\left(X_{k}, x_{k}, g_{k}(0)\right) \stackrel{\text { Gromov-Hausdorff }}{\longrightarrow}\left(X_{\infty}, x_{\infty}, g_{\infty}\right) .
$$

Combining (25), (26), and (27), we obtain a locally Lipschitz limit function $\varphi_{\infty}$ on $B\left(x_{\infty}, 1\right) \subset X_{\infty}$ with $\left\|\varphi_{\infty}\right\|_{C^{0}\left(B\left(x_{\infty}, 1\right)\right)} \leq C_{1}$. In general, it is hard to expect $\varphi_{\infty}$ to be better than a locally Lipschitz function. However, by Theorem 0.8 of [19], we know that $X_{\infty}$ is isometric to the Euclidean space $\left(\mathbb{R}^{m}, g_{\mathbb{E}}\right)$, which has a lot of excellent properties. We will use these properties to show that $\varphi_{\infty}$ has much better regularity than a general locally Lipschitz function. 
Claim 1. $\varphi_{\infty}$ can be extended to be a continuous function defined on $\overline{B\left(x_{\infty}, 1\right)}$ with

$$
\left.\varphi_{\infty}\right|_{\partial B\left(x_{\infty}, 1\right)}=0
$$

It suffices to show $\lim _{r \rightarrow 0}\left\|\varphi_{\infty}\right\|_{L^{\infty}(B(w, r))}=0$ for arbitrary $w \in \partial B\left(x_{\infty}, 1\right)$.

Fix arbitrary $w \in \partial B\left(x_{\infty}, 1\right)$. Suppose $w_{k} \in \partial B\left(x_{k}, 1\right)$ and $w_{k} \rightarrow w$ as $X_{k}$ converges to $X_{\infty}$. For brevity, define $M_{d, k} \triangleq O s c_{B\left(w_{k}, d\right)}\left(\varphi_{k}\right)$. By trivial extension, we can look at $\varphi_{k}$ as a function defined on the whole manifold $X_{k}$, which is a subsolution of (23) in the sense of distribution. Define $\psi_{d, k} \triangleq M_{2 d, k}-\varphi_{k}$, and then it is easy to see that $\psi_{d, k}$ satisfies the inequality

$$
\begin{aligned}
& \left(-\Delta-\frac{1}{2}\left(m+m \log \sqrt{2 \pi}+\lambda_{k}\right)\right) \psi_{d, k} \\
& =-\frac{M_{2 d, k}\left(m+m \log \sqrt{2 \pi}+\lambda_{k}\right)}{2}-\left(M_{2 d, k}-\psi_{d, k}\right) \log \left(M_{2 d, k}-\psi_{d, k}\right) \\
& \geq-C_{3}=-C_{3}\left(m, C_{S}, C_{\lambda}\right)
\end{aligned}
$$

in the sense of distribution. In other words, $\psi_{d, k}$ is a super-solution of the corresponding elliptic equation. Clearly, in the ball $B\left(w_{k}, 4 d\right) \subset B\left(x_{k}, 10\right)$, every geodesic ball's volume ratio is bounded from two sides. Applying Proposition 2.2. we obtain

$$
(2 d)^{-m} \int_{B\left(w_{k}, 2 d\right)} \psi_{d, k} \leq C_{4}\left(\inf _{B\left(w_{k}, d\right)} \psi_{d, k}+d^{2}\right)
$$
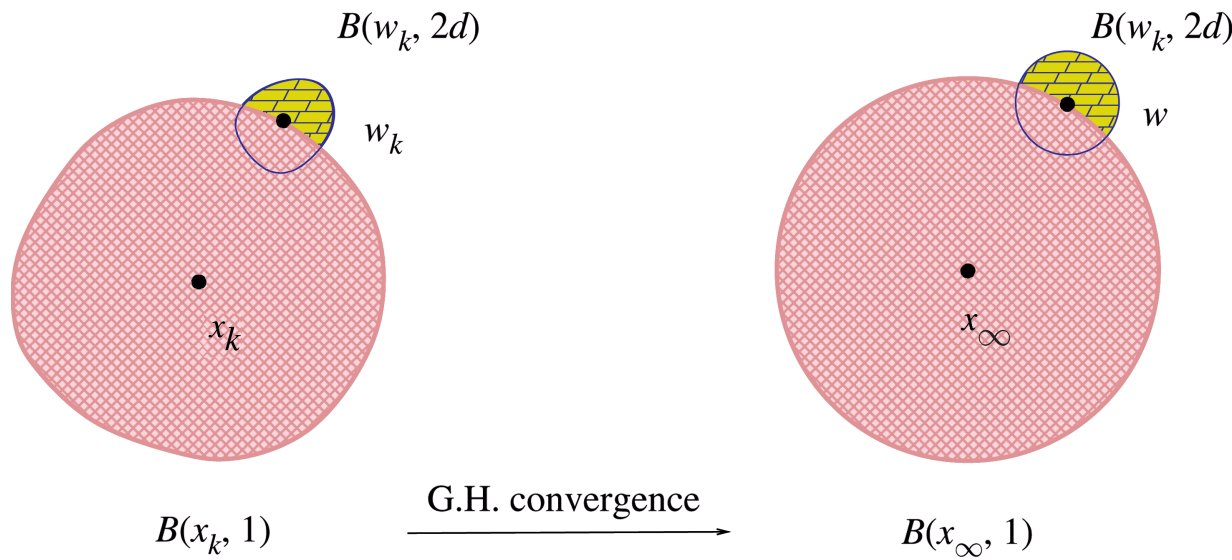

FiguRE 1. Boundary estimates

By the volume continuity, it is not hard (Figure 1) to see that the volume of $B\left(w_{k}, 2 d\right) \backslash B\left(x_{k}, 1\right)$ is strictly greater than a fixed portion of the volume of $B\left(w_{k}, 2 d\right)$, which is almost $\omega_{m}(2 d)^{m}$. For brevity, let us say $\left|B\left(w_{k}, 2 d\right) \backslash B\left(x_{k}, 1\right)\right|>$ $10^{-m} \cdot \omega_{m}(2 d)^{m}$. Putting this into (30) and noting that $\inf _{B\left(w_{k}, d\right)} \psi_{d, k}=M_{2 d, k}-M_{d, k}$, we have $10^{-m} \omega_{m} M_{2 d, k}<C_{4}\left(M_{2 d, k}-M_{d, k}+d^{2}\right)$, which implies

$$
M_{d, k}<\left(1-10^{-m} C_{4}^{-1} \omega_{m}\right) M_{2 d, k}+d^{2} \triangleq \gamma M_{2 d, k}+d^{2} .
$$


By choosing $C_{4}$ large, we can assume $\gamma \in\left(\frac{3}{4}, 1\right)$. Let $d=2^{-i}, i>1$. Induction of (31) yields

$M_{2^{-i}, k}<\gamma M_{2^{-i+1}, k}+4^{-i}<\gamma^{i-1} M_{\frac{1}{2}, k}+\sum_{j=0}^{i-2} \gamma^{j} 4^{-i+j}=\gamma^{i-1} M_{\frac{1}{2}, k}+\frac{\gamma^{i-1}-4^{-i+1}}{4(4 \gamma-1)}$.

Recall that $M_{\frac{1}{2}, k} \leq\left\|\varphi_{k}\right\|_{C^{0}\left(B\left(x_{k}, 1\right)\right)} \leq C_{1}$. Letting $k \rightarrow \infty$, we obtain

$$
\left\|\varphi_{\infty}\right\|_{L^{\infty}\left(B\left(w, 2^{-i}\right)\right)} \leq \lim _{k \rightarrow \infty} M_{2^{-i+1}, k} \leq C_{1} \gamma^{i-1}+\frac{\gamma^{i-1}-4^{-i+1}}{4(4 \gamma-1)}<C \gamma^{i},
$$

where $C=C(m)$. Since $\gamma<1$, it is clear that (32) implies $\lim _{r \rightarrow 0}\left\|\varphi_{\infty}\right\|_{L^{\infty}(B(w, r))}=0$. So we finish the proof of Claim 1 .

The proof of Claim 1 originates from the boundary estimate of Section 8.10 of [25. Actually, by the arbitrary choice of $w \in \partial B\left(x_{\infty}, 1\right)$ and compactness, for arbitrary $\eta \in\left(0, \frac{1}{2}\right)$, inequality (32) implies

$$
\left\|\varphi_{\infty}\right\|_{L^{\infty}\left(B\left(x_{\infty}, 1\right) \backslash B\left(x_{\infty}, 1-\eta\right)\right)}<C \eta^{\alpha},
$$

where $C=C(m)$ and $\alpha=-\frac{\log \gamma}{\log 2} \in(0,1)$. In other words, the function $\varphi_{\infty}$ satisfies the boundary $C^{\alpha}$-estimate.

Claim 2. In $B\left(x_{\infty}, 1\right), \varphi_{\infty}$ satisfies the following equation:

$$
-2 \Delta \varphi_{\infty}-2 \varphi_{\infty} \log \varphi_{\infty}-\left(m+m \log \sqrt{2 \pi}+\lambda_{\infty}\right) \varphi_{\infty}=0 .
$$

Consequently, $\varphi_{\infty} \in C^{\infty}\left(B\left(x_{\infty}, 1\right)\right)$.

Note that $B\left(x_{\infty}, 1\right)$ is a unit ball in the standard $\mathbb{R}^{m}$. In particular, it has a smooth boundary. So Equation (34) is equivalent to the following integration equation:

$$
\varphi_{\infty}(z)=\int_{B\left(x_{\infty}, 1\right)} G(z, y)\left(\frac{m+m \log \sqrt{2 \pi}+\lambda_{\infty}}{2}+\log \varphi_{\infty}(y)\right) \varphi_{\infty}(y) d y,
$$

for every $z \in B\left(x_{\infty}, 1\right)$. Here $G$ is the Green function of the unit ball $B\left(x_{\infty}, 1\right) \subset$ $\mathbb{R}^{m}$. Because $B\left(x_{\infty}, 1\right)$ is simple, we can write down $G(z, y)$ explicitly,

$$
G(z, y)=\frac{1}{(m-2) m \omega_{m}}\left(d^{2-m}(z, y)-d^{2-m}\left(x_{\infty}, z\right) d^{2-m}\left(z^{*}, y\right)\right),
$$

whenever $z \neq y$. Here $z^{*}$ is the symmetric point of $z$ with respect to $\partial B\left(x_{\infty}, 1\right)$. If $z \neq x_{\infty}, z^{*}$ is the point such that $x_{\infty}, z, z^{*}$ on the same straight line and $\left|\overline{x_{\infty} z}\right|$. $\left|\overline{x_{\infty} z^{*}}\right|=1$. If $z=x_{\infty}$, we assume $z^{*}$ as the infinity point. In the later case, we have

$$
G\left(x_{\infty}, y\right)=\frac{1}{(m-2) m \omega_{m}}\left(d^{2-m}\left(x_{\infty}, y\right)-1\right) .
$$

By continuity, for proving (35) in $B\left(z_{\infty}, 1\right)$, it suffices to show (35) for every $z \in B\left(x_{\infty}, 1\right) \backslash\left\{x_{\infty}\right\}$. Without loss of generality, we fix an arbitrary point $z \in$ $B\left(x_{\infty}, 1\right) \backslash\left\{x_{\infty}\right\}$. Suppose $z_{k} \in B\left(x_{k}, 1\right)$ and $z_{k} \rightarrow z, z_{k}^{*} \in X_{k}$ and $z_{k}^{*} \rightarrow z^{*}$ (see Figure 2).

Let $d$ be the distance function to the point $z_{k}$ under the metric $g_{k}(0)$. Note that

$$
\Delta d^{2-m}=(2-m) d^{-m}(1-m+d \Delta d) .
$$




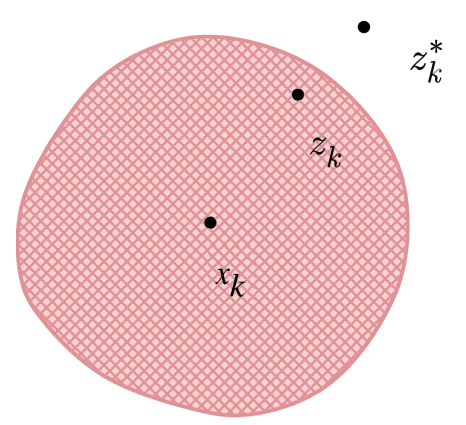

$B\left(x_{k}, 1\right)$ $z_{k}^{*}$

\section{G.H. convergence}

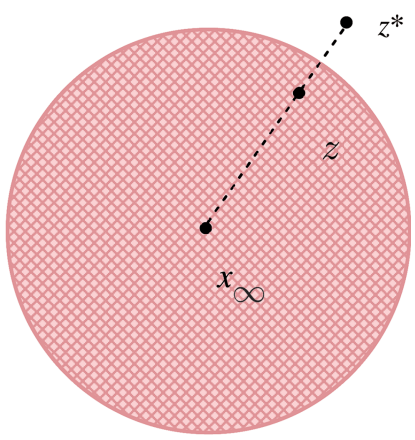

$B\left(x_{\infty}, 1\right)$

Figure 2. Approximation of Green functions

If the underlying space is Euclidean, then the right hand side is equal to 0 whenever $d>0$. Now on $X_{k}$, we are focusing our attention around the point $x_{k}$, where Ric $\geq-(m-1) \delta_{k}^{4}$. Clearly, the Laplacian comparison theorem (cf. Corollary 1.131 of [14]) implies that

$$
\Delta d^{2-m}+(m-2)(m-1) d^{1-m} \delta_{k}^{2} \geq 0
$$

on $B\left(x_{k}, 10\right)$. It follows that

$$
\begin{aligned}
0 \leq & \int_{B\left(x_{k}, 1\right) \backslash B\left(z_{k}, r\right)}\left\{\Delta d^{2-m}+(m-2)(m-1) d^{1-m} \delta_{k}^{2}\right\} \\
\leq & \int_{B\left(z_{k}, 2\right) \backslash B\left(z_{k}, r\right)}\left\{\Delta d^{2-m}+(m-2)(m-1) d^{1-m} \delta_{k}^{2}\right\} \\
= & (m-2)\left\{\left|\partial B\left(z_{k}, 2\right)\right| 2^{1-m}-\left|\partial B\left(z_{k}, r\right)\right| r^{1-m}\right\} \\
& +(m-2)(m-1) \delta_{k}^{2} \int_{r}^{2}\left(\rho^{1-m}\left|\partial B\left(z_{k}, \rho\right)\right|\right) d \rho \\
< & (m-2)\left\{|| \partial B\left(z_{k}, 2\right)\left|2^{1-m}-\right| \partial B\left(z_{k}, r\right)\left|r^{1-m}\right|+4(m-1) m \omega_{m} \delta_{k}^{2}\right\} .
\end{aligned}
$$

Consequently, we have

$$
\begin{aligned}
& \int_{B\left(x_{k}, 1\right) \backslash B\left(z_{k}, r\right)}\left|\Delta d^{2-m}\right| \\
& \quad \leq \int_{B\left(x_{k}, 1\right) \backslash B\left(z_{k}, r\right)}\left|\Delta d^{2-m}+(m-2)(m-1) d^{1-m} \delta_{k}^{2}\right| \\
& \quad+\int_{B\left(x_{k}, 1\right) \backslash B\left(z_{k}, r\right)}(m-2)(m-1) d^{1-m} \delta_{k}^{2} \\
& \quad<(m-2)\left\{|| \partial B\left(z_{k}, 2\right)\left|2^{1-m}-\right| \partial B\left(z_{k}, r\right)\left|r^{1-m}\right|+8(m-1) m \omega_{m} \delta_{k}^{2}\right\} .
\end{aligned}
$$

Fix $k$, let $r \rightarrow 0$, and we have

$$
\int_{B\left(x_{k}, 1\right) \backslash\left\{z_{k}\right\}}\left|\Delta d^{2-m}\right| \leq(m-2)\left\{|| \partial B\left(z_{k}, 2\right)\left|2^{1-m}-m \omega_{m}\right|+8(m-1) m \omega_{m} \delta_{k}^{2}\right\} .
$$


Therefore, we obtain

$$
\begin{aligned}
\int_{B\left(x_{k}, 1\right) \backslash\left\{z_{k}\right\}}\left|\varphi_{k} \Delta d^{2-m}\right| & \leq C_{1}(m-2)\left\{|| \partial B\left(z_{k}, 2\right)\left|2^{1-m}-m \omega_{m}\right|\right. \\
\left.+8(m-1) m \omega_{m} \delta_{k}^{2}\right\} & \rightarrow 0,
\end{aligned}
$$

as $k \rightarrow \infty$, since the limit space $X_{\infty}$ is Euclidean, where every geodesic sphere has the same volume ratio: $m \omega_{m}$.

From Green's representation formula in $\mathbb{R}^{m}$, one can expect the following equation to hold:

$$
\begin{aligned}
(2-m) m \omega_{m} \varphi_{\infty}(z)= & \lim _{k \rightarrow \infty} \int_{B\left(x_{k}, 1\right)}\left\{d^{2-m}\left(z_{k}, y\right)-d^{2-m}\left(x_{k}, z_{k}\right) d^{2-m}\left(z_{k}^{*}, y\right)\right\} \\
& \Delta \varphi_{k}(y) d y .
\end{aligned}
$$

Actually, (39) can be proved as follows. Fixing each positive $\eta<\frac{1}{2}\left(1-\left|\overline{x_{\infty} z}\right|\right)$, we can calculate

$$
\begin{aligned}
& \int_{B\left(x_{k}, 1-\eta\right)} d^{2-m}\left(z_{k}, y\right) \Delta \varphi_{k}(y) d y \\
& =\lim _{r \rightarrow 0} \int_{B\left(x_{k}, 1-\eta\right) \backslash B\left(z_{k}, r\right)} d^{2-m}\left(z_{k}, y\right) \Delta \varphi_{k}(y) d y \\
& =\int_{B\left(x_{k}, 1-\eta\right) \backslash\left\{z_{k}\right\}} \varphi_{k}(y) \Delta d^{2-m}\left(z_{k}, y\right) d y-(m-2) m \omega_{m} \varphi_{k}\left(z_{k}\right) \\
& \quad+\int_{\partial B\left(x_{k}, 1-\eta\right)}\left(d^{2-m}\left(z_{k}, y\right) \nabla \varphi_{k}-\varphi_{k} \nabla d^{2-m}\left(z_{k}, y\right)\right) \cdot \vec{n} d \sigma_{y} .
\end{aligned}
$$

Here $d y=d \mu_{g_{k}(0)}$, and $d \sigma_{y}$ is the induced measure on $\partial B\left(x_{k}, 1-\eta\right)$ by the metric $g_{k}(0)$. In view of a bound similar to (38), we obtain

$$
\begin{aligned}
& \lim _{k \rightarrow \infty} \int_{B\left(x_{k}, 1-\eta\right)} d^{2-m}\left(z_{k}, y\right) \Delta \varphi_{k}(y) d y=(2-m) m \omega_{m} \varphi_{\infty}\left(z_{\infty}\right) \\
& \quad+\lim _{k \rightarrow \infty} \int_{\partial B\left(x_{k}, 1-\eta\right)}\left(d^{2-m}\left(z_{k}, y\right) \nabla \varphi_{k}-\varphi_{k} \nabla d^{2-m}\left(z_{k}, y\right)\right) \cdot \vec{n} d \sigma_{y} .
\end{aligned}
$$

Note that $d\left(z_{k}^{*}, \cdot\right)>0$ uniformly on $B\left(x_{k}, 1\right)$. By similar but simpler arguments, we obtain

$$
\begin{aligned}
& \lim _{k \rightarrow \infty} \int_{B\left(x_{k}, 1-\eta\right)} d^{2-m}\left(z_{k}^{*}, y\right) \Delta \varphi_{k}(y) d y \\
& \quad=\lim _{k \rightarrow \infty} \int_{\partial B\left(x_{k}, 1-\eta\right)}\left(d^{2-m}\left(z_{k}^{*}, y\right) \nabla \varphi_{k}-\varphi_{k} \nabla d^{2-m}\left(z_{k}^{*}, y\right)\right) \cdot \vec{n} d \sigma_{y} .
\end{aligned}
$$

Combining (41) and (42) yields that

$$
\begin{aligned}
\lim _{k \rightarrow \infty} & \int_{B\left(x_{k}, 1-\eta\right)}\left(d^{2-m}\left(z_{k}, y\right)-d^{2-m}\left(x_{k}, z_{k}\right) d^{2-m}\left(z_{k}^{*}, y\right)\right) \\
& \Delta \varphi_{k}(y) d y+(m-2) m \omega_{m} \varphi_{\infty}\left(z_{\infty}\right) \\
= & \int_{\partial B\left(x_{k}, 1-\eta\right)}\left\{\left(d^{2-m}\left(z_{k}, y\right)-d^{2-m}\left(z_{k}, x_{k}\right) d^{2-m}\left(z_{k}^{*}, y\right)\right) \nabla \varphi_{k}\right. \\
& \left.-\varphi_{k} \nabla\left(d^{2-m}\left(z_{k}, y\right)-d^{2-m}\left(z_{k}, x_{k}\right) d^{2-m}\left(z_{k}^{*}, y\right)\right)\right\} \cdot \vec{n} d \sigma_{y} .
\end{aligned}
$$


Now we estimate the right hand side of the above inequality. First, we note that on $\partial B\left(x_{k}, 1-\eta\right)$, we have $\left|\varphi_{k}\right|<C(m) \eta^{\alpha}$, in light of (33). Second, by a Cheng-Yau type estimate, we also have

$$
\left|\nabla \varphi_{k}\right|<C \eta^{\alpha-1}
$$

for some $C=C(m)$. This can be done from either the maximum principle or the Moser iteration. Here we sketch a maximum principle argument, following Theorem 7.1 of Cheeger's note 11. Note that by replacing a constant, we only need to show (44) on $\partial B\left(x_{k}, 1-2 \eta\right)$. Let $u$ be one of $\varphi_{k}$, and $e$ be a point on $\partial B\left(x_{k}, 1-2 \eta\right)$. Then $u$ satisfies the equation

$$
\Delta u=-A u-u \log u
$$

for some uniformly bounded constant $A$ in the ball $B(e, \eta)$ where $|u|<C \eta^{\alpha}$ for some uniformly constant $C$. Let $\chi$ be a non-increasing smooth cutoff function defined on $[0,1]$ such that $\chi \equiv 1$ on $[0,0.5]$ and vanishes around 1 , to be determined later. By abuse of notation, we also denote the radial cutoff function $\chi\left(\frac{r(\cdot, e)}{\eta}\right)$ by $\chi$, where $r$ is the distance to point $e$. Clearly, $\chi$ vanishes on the boundary $\partial B(e, \eta)$ and equals 1 on $B(e, 0.5 \eta)$. Calculating $\Delta\left(\chi|\nabla \log u|^{2}\right)$ and applying the maximum principle, as done in [11, we obtain

$|\nabla \log u|^{2} \leq \max \left\{-2(A+\log u), 4 m\left(-\frac{\Delta \chi}{\chi}+(4 m+2) \frac{|\nabla \chi|^{2}}{\chi^{2}}+2(m-1)+\frac{2}{u}\right)\right\}$.

Note that $-\Delta \chi=-\nabla\left(\chi^{\prime} \frac{\nabla r}{\eta}\right)=-\eta^{-2} \chi^{\prime \prime}-\eta^{-1} \chi^{\prime} \Delta r$ and $|\nabla \chi|^{2}=\eta^{-2}\left|\chi^{\prime}\right|^{2}$. Note also that $\chi^{\prime} \leq 0$ and vanishes on $[0,0.5]$, and $r \Delta r \leq m$ since we choose $\eta$ very small. So we have $-\eta \chi^{\prime} \Delta r=0$ whenever $0 \leq r \leq 0.5 \eta$ and $-\eta \chi^{\prime} \Delta r \leq-\chi^{\prime} \frac{m \eta}{r} \leq-2 m \chi^{\prime}$ whenever $0.5 \eta \leq r \leq \eta$. It follows that

$$
\begin{aligned}
-\frac{\Delta \chi}{\chi}+(4 m+2) \frac{|\nabla \chi|^{2}}{\chi^{2}} & =-\eta^{-2} \chi^{-1}\left(\chi^{\prime \prime}+\eta \chi^{\prime} \Delta r-(4 m+2) \eta^{2} \chi^{-1}\left|\chi^{\prime}\right|^{2}\right) \\
& \leq-\eta^{-2} \chi^{-1}\left(\chi^{\prime \prime}+2 m \chi^{\prime}-(4 m+2) \eta^{2} \chi^{-1}\left|\chi^{\prime}\right|^{2}\right) \\
& \leq-\eta^{-2} \chi^{-1}\left(\chi^{\prime \prime}+2 m \chi^{\prime}+\frac{1}{100} \chi^{-1}\left|\chi^{\prime}\right|^{2}\right) \\
& \leq C \eta^{-2}
\end{aligned}
$$

since we can choose a cutoff function $\chi$ on $[0,1]$ such that $\chi^{\prime \prime}+2 m \chi^{\prime}+\frac{1}{100} \chi^{-1}\left|\chi^{\prime}\right|^{2}>$ 0 on $(1-\epsilon, 1)$ for a very small $\epsilon$. For example, let $\chi(t)=e^{\frac{1}{t-1}}$ around 1. Plugging the estimate $|u|<C \eta^{\alpha}$ into the Cheng-Yau estimate of $|\nabla \log u|^{2}$, we have

$$
|\nabla \log u|^{2}<C \eta^{-2} \text {, in } B(e, 0.5 \eta),
$$

and consequently $|\nabla u|<C \eta^{\alpha-1}$ in $B(e, 0.5 \eta)$. Recall that $u$ is one of $\varphi_{k}$. So we have proved (44). Third, it is not hard to see that on $\partial B\left(x_{k}, 1-\eta\right)$ we have

$$
\begin{aligned}
& \left|d^{2-m}\left(z_{k}, y\right)-d^{2-m}\left(z_{k}, x_{k}\right) d^{2-m}\left(z_{k}^{*}, y\right)\right|<C \eta, \\
& \quad\left|\nabla\left(d^{2-m}\left(z_{k}, y\right)-d^{2-m}\left(z_{k}, x_{k}\right) d^{2-m}\left(z_{k}^{*}, y\right)\right)\right|<C
\end{aligned}
$$


for some $C=C\left(m,\left|\overline{x_{\infty} z}\right|\right)$. Plugging all the three ingredients mentioned above into (43), we have

$$
\begin{gathered}
\mid \lim _{k \rightarrow \infty} \int_{B\left(x_{k}, 1-\eta\right)}\left(d^{2-m}\left(z_{k}, y\right)-d^{2-m}\left(x_{k}, z_{k}\right) d^{2-m}\left(z_{k}^{*}, y\right)\right) \\
\Delta \varphi_{k}(y) d y+(m-2) m \omega_{m} \varphi_{\infty}\left(z_{\infty}\right) \mid<C \eta^{\alpha},
\end{gathered}
$$

where $C=C\left(m,\left|\overline{x_{\infty} z}\right|\right)$. Letting $\eta \rightarrow 0$, we obtain (39).

Plugging the Euler-Lagrange equation (23) into (39), we obtain

$$
\begin{aligned}
(m-2) m \omega_{m} \varphi_{\infty}(z) & \\
= & \lim _{k \rightarrow \infty} \int_{B\left(x_{k}, 1\right)}\left\{d^{2-m}\left(z_{k}, y\right)-d^{2-m}\left(x_{k}, z_{k}\right) d^{2-m}\left(z_{k}^{*}, y\right)\right\} \\
& \left(\frac{m+m \log \sqrt{2 \pi}+\lambda_{k}}{2}+\log \varphi_{k}(y)\right) \varphi_{k}(y) d y .
\end{aligned}
$$

The right hand side of the above equation can be simplified. Actually, if we denote

$$
\begin{aligned}
H_{k}=\{ & \left.d^{2-m}\left(z_{k}, y\right)-d^{2-m}\left(x_{k}, z_{k}\right) d^{2-m}\left(z_{k}^{*}, y\right)\right\} \\
& \left(\frac{m+m \log \sqrt{2 \pi}+\lambda_{k}}{2}+\log \varphi_{k}(y)\right) \varphi_{k}(y), \\
H_{\infty}= & \left\{d^{2-m}(z, y)-d^{2-m}\left(x_{\infty}, z\right) d^{2-m}\left(z^{*}, y\right)\right\} . \\
& \left(\frac{m+m \log \sqrt{2 \pi}+\lambda_{\infty}}{2}+\log \varphi_{\infty}(y)\right) \varphi_{\infty}(y),
\end{aligned}
$$

then for each $\epsilon>0$, in light of the volume convergence and uniform convergence of $\varphi_{k}$ 2 (cf. inequality (25), (26), or (44)), we have

$$
\lim _{k \rightarrow \infty} \int_{B\left(x_{k}, 1-\epsilon\right) \backslash B\left(z_{k}, \epsilon\right)} H_{k}(y) d y=\int_{B\left(x_{\infty}, 1-\epsilon\right) \backslash B\left(z_{\infty}, \epsilon\right)} H_{\infty}(y) d y .
$$

Note that $\left|B\left(x_{k}, 1\right) \backslash\left\{B\left(x_{k}, 1-\epsilon\right) \cup B\left(z_{k}, \epsilon\right)\right\}\right| \leq C \epsilon$ for some uniform $C$, due to Bishop-Gromov volume comparison. Letting $\epsilon \rightarrow 0$, we obtain

$$
\begin{aligned}
\lim _{k \rightarrow \infty} \int_{B\left(x_{k}, 1\right)} H_{k}(y) d y= & \int_{B\left(x_{\infty}, 1\right)} H_{\infty}(y) d y=(m-2) m \omega_{m} \int_{B\left(x_{\infty}, 1\right)} G(z, y) . \\
& \left(\frac{m+m \log \sqrt{2 \pi}+\lambda_{\infty}}{2}+\log \varphi_{\infty}(y)\right) \varphi_{\infty}(y) d y
\end{aligned}
$$

Therefore, we have proved (35) for $z$ in light of (45) and (46). By the arbitrariness of $z \in B\left(x_{\infty}, 1\right) \backslash\left\{x_{\infty}\right\}$ and continuity, Equation (35), henceforth (34), follows directly. Then the standard bootstrapping argument for elliptic PDEs implies that $\varphi_{\infty} \in C^{\infty}\left(B\left(x_{\infty}, 1\right)\right)$. This finishes the proof of Claim 2.

Now we are ready to prove the theorem by a contradiction argument. Since $\varphi_{\infty}$ is a bounded smooth function satisfying the differential equation (34) and the boundary condition $\left.\varphi_{\infty}\right|_{\partial B\left(x_{\infty}, 1\right)} \equiv 0$, one can obtain $\left\|\nabla \varphi_{\infty}\right\|_{L^{2}\left(B\left(x_{\infty}, 1\right)\right)} \leq C$ from

\footnotetext{
${ }^{2}$ For more details, interested readers can refer to Equation (2.13) of [7] and the discussion above it.
} 
integration by parts on the level sets of $\varphi_{\infty}$. Note that $B\left(x_{\infty}, 1\right)$ is a bounded domain with smooth boundary, $\left.\varphi_{\infty}\right|_{\partial B\left(x_{\infty}, 1\right)} \equiv 0$, and by trivial extension, we can regard $\varphi_{\infty} \in W_{0}^{1,2}\left(\mathbb{R}^{m}\right)$ (cf. Section 5.5 of [21]). It follows from the Logarithm Sobolev inequality of Euclidean space (cf. 24]) that

$$
\int_{\mathbb{R}^{m}}\left(\frac{1}{2}\left|\nabla \varphi_{\infty}\right|^{2}-2 \varphi_{\infty}^{2} \log \varphi_{\infty}-m(1+\log \sqrt{2 \pi}) \varphi_{\infty}^{2}\right) \geq 0 .
$$

On the other hand, by (34) in Claim 2 and the fact $\varphi_{\infty} \equiv 0$ outside $B\left(x_{\infty}, 1\right)$, we deduce that

$$
\int_{\mathbb{R}^{m}}\left(\frac{1}{2}\left|\nabla \varphi_{\infty}\right|^{2}-2 \varphi_{\infty}^{2} \log \varphi_{\infty}-m(1+\log \sqrt{2 \pi}) \varphi_{\infty}^{2}\right)=\lambda_{\infty} \leq-\eta<0,
$$

which contradicts to (47)!

Remark 3.1. If the "almost-Euclidean volume ratio" (inequality (12)) and "almost nonnegative Ricci" (inequality (11)) hold globally, then the rough curvature estimate (inequality (14)) follows from the combination of Perelman's pseudo-locality theorem and Levy-Gromov inequality (cf [23]), whose proof requires some regularity results in geometric measure theory on closed manifolds. There should exist another proof of Proposition 3.1 from some local version of the Levy-Gromov inequality. However, it seems that some local regularity results in geometric measure theory are required.

Remark 3.2. Except inequality (11), the "almost non-negative Ricci" condition can also be interpreted as the $L^{p}$-integration of the negative Ricci part and is sufficiently small ( $c f$ 31, 32]), for some $p>\frac{m}{2}$. Using this interpretation, one can obtain another pseudo-locality theorem.

Combining Proposition 3.1 with the fundamental work of [6], we obtain the following property.

Proposition 3.2. There exists a constant $\delta_{0}=\delta_{0}(m)$ with the following properties.

Suppose $\{(X, g(t)), 0 \leq t \leq 1\}$ is a Ricci flow solution, $x_{0} \in X, \Omega=B_{g(0)}\left(x_{0}, 1\right)$. Suppose that

$$
\operatorname{Ric}(x, 0) \geq-(m-1) \delta_{0}, \quad \forall x \in X ; \quad|\Omega|_{d \mu_{g(0)}} \geq\left(1-\delta_{0}\right) \omega_{m} .
$$

Then for every $x \in \Omega^{\prime}=B_{g(0)}\left(x_{0}, \frac{3}{4}\right), s \in\left(0,2 \delta_{0}\right]$, we have

$$
\left|B_{g(s)}(x, \sqrt{s})\right|_{d \mu_{g(s)}} \geq \kappa^{\prime} s^{\frac{m}{2}}, \quad \sup _{B_{g(s)}(x, \sqrt{s})}|R m|(\cdot, s) \leq \frac{1}{100} s^{-1},
$$

where $\kappa^{\prime}=\kappa^{\prime}(m)$ is a universal constant.

Proof. Let us first prove the following Claim.

Claim 3. For every small $\xi>0$, there exists a number $\eta=\eta(m, \xi)$ with the following property. then

Suppose $\operatorname{Ric}(x, 0) \geq-(m-1) \eta$ in $\Omega=B_{g(0)}\left(x_{0}, 1\right)$, and $|\Omega|_{d \mu_{g(0)}} \geq(1-\eta) \omega_{m}$,

$$
8^{m}\left|B_{g(0)}\left(y, \frac{1}{8}\right)\right|_{d \mu_{g(0)}} \geq(1-\xi) \omega_{m}, \quad \forall y \in B_{g(0)}\left(x_{0}, \frac{3}{4}\right)
$$


Actually, if this statement was wrong, we can find a sequence of $\eta_{i} \rightarrow 0$ and manifolds $\left(X_{i}, x_{i}, g_{i}(0)\right)$ such that (48) holds for $\eta_{i}$ and the ball $\Omega_{i}=B_{g_{i}(0)}\left(x_{i}, 1\right)$. However, for some point $y_{i} \in B_{g_{i}(0)}\left(x_{i}, \frac{3}{4}\right)$, we have

$$
8^{m}\left|B_{g_{i}(0)}\left(y_{i}, \frac{1}{8}\right)\right|_{d \mu_{g_{i}(0)}}<(1-\xi) \omega_{m}
$$

Suppose $\left(\Omega_{i}, x_{i}, g_{i}(0)\right)$ converges to $(\bar{\Omega}, \bar{x}, \bar{g})$. By Theorem 0.8 of [19], we see that $\bar{\Omega}$ is isometric to the unit ball in the Euclidean space $\mathbb{R}^{m}$. Since $y_{i} \in B_{g_{i}(0)}\left(x_{i}, \frac{3}{4}\right)$, we can assume $y_{i} \rightarrow \bar{y} \in B\left(\bar{x}, \frac{3}{4}+\frac{1}{100}\right) \subset \bar{\Omega}$. The lower bound of Ricci guarantees the continuity of volume (cf. Theorem 0.1. of [19]). Therefore we have

$$
\lim _{i \rightarrow \infty} 8^{m}\left|B_{g_{i}(0)}\left(y_{i}, \frac{1}{8}\right)\right|_{d \mu_{g_{i}(0)}}=8^{m}\left|B_{\bar{g}}\left(\bar{y}, \frac{1}{8}\right)\right|_{d \mu_{\bar{g}}}=\omega_{m}
$$

which contradicts to (50)! This contradiction establishes the proof of Claim 3 .

Let $\xi=\delta^{4}\left(m, \frac{1}{1000 m}\right)$, where $\delta$ is defined by Proposition 3.1. Let $\eta=\eta(m, \xi)$ according to Claim 3 .

Suppose the conditions of Claim 3 are satisfied for $\eta=\eta(m, \xi)$. Define $\hat{g}(t)=$ $\xi^{-2} g\left(\xi^{2} t\right)$. Fix an arbitrary point $y \in B_{g(0)}\left(x_{0}, \frac{3}{4}\right)$. By volume comparison, inequality (49) and the choice of $\xi$ yield that $(X, y, \hat{g}(0))$ satisfies the initial conditions of Proposition 3.1. In particular, we have $\left|B_{\hat{g}(t)}(y, \sqrt{t})\right|_{d \mu_{\hat{g}(t)}} \geq \kappa^{\prime} t^{\frac{m}{2}}, \quad \sup _{B_{\hat{g}(t)}(y, \sqrt{t})}$ $|R m|_{\hat{g}(t)}(\cdot) \leq \frac{1}{1000 m t}+\epsilon^{-2}$ for every $t \in\left(0, \epsilon^{2}\right]$. This implies that for every $t \in\left(0, \frac{\epsilon^{2}}{200}\right]$, we have

$$
\left|B_{\hat{g}(t)}(y, \sqrt{t})\right|_{d \mu_{\hat{g}(t)}} \geq \kappa^{\prime} t^{\frac{m}{2}}, \quad \sup _{B_{\hat{g}(t)}(y, \sqrt{t})}|R m|_{\hat{g}(t)}(\cdot) \leq \frac{1}{100 t} .
$$

By a trivial rescaling argument, we conclude

$$
\left|B_{g(t)}(y, \sqrt{t})\right|_{d \mu_{g(t)}} \geq \kappa^{\prime} t^{\frac{m}{2}}, \quad \sup _{B_{g(t)}(y, \sqrt{t})}|R m|_{\hat{g}(t)}(\cdot) \leq \frac{1}{100 t}, \quad \forall t \in\left(0, \frac{\xi^{2} \epsilon^{2}}{200}\right] .
$$

Define $\delta_{0} \triangleq \min \left\{\frac{\xi^{2} \epsilon^{2}}{1000}, \eta(m, \xi)\right\}$. Clearly, Proposition 3.2 holds for this choice of $\delta_{0}$.

Now we are ready to prove the pseudo-locality theorem under the normalized Ricci flow.

Theorem 3.1 (Pseudo-locality theorem). There exists a constant $\delta_{0}=\delta_{0}(\mathrm{~m})$ with the following properties.

Suppose $\{(X, g(t)), 0 \leq t \leq 1\}$ is a normalized Ricci flow solution: $\frac{\partial}{\partial t} g=-$ Ric + $\lambda_{0} g$, and $\lambda_{0}$ is a constant with $\left|\lambda_{0}\right| \leq 1$. Let $x_{0} \in X, \Omega=B_{g(0)}\left(x_{0}, 1\right)$. Suppose that

$$
\operatorname{Ric}(x, 0) \geq-(m-1) \delta_{0}, \quad \forall x \in X ; \quad|\Omega|_{d \mu_{g(0)}} \geq\left(1-\delta_{0}\right) \omega_{m} .
$$

Then we have

$$
\begin{aligned}
& \left|B_{g(t)}(x, \sqrt{t})\right|_{d \mu_{g(t)}} \geq \kappa_{0} t^{\frac{m}{2}}, \\
& \sup _{B_{g(t)}(x, \sqrt{t})}|R m|(x, t) \leq t^{-1}, \quad \forall x \in \Omega^{\prime}=B_{g(0)}\left(x_{0}, \frac{3}{4}\right), t \in\left(0,2 \delta_{0}\right],
\end{aligned}
$$

where $\kappa_{0}=\kappa_{0}(m)$ is a universal constant. 
Proof. Let $\tilde{g}(s)=\left(1-2 \lambda_{0} s\right) g\left(\frac{\log \left(1-2 \lambda_{0} s\right)}{-\lambda_{0}}\right)$. Clearly, $\tilde{g}(s)$ is a Ricci flow solution with $\tilde{g}(0)=g(0)$. Denote $\frac{\log \left(1-2 \lambda_{0} s\right)}{-\lambda_{0}}$ by $t(s)$. Then we have $\tilde{g}(s)=\left(1-2 \lambda_{0} s\right) g(t)$. By Taylor expansion of $t(s)=\frac{\log \left(1-2 \lambda_{0} s\right)}{-\lambda_{0}}$, shrinking $\delta_{0}$ if necessary, we have $\frac{3}{2} s<$ $t<3 s$ whenever $s \in\left(0,10 \delta_{0}\right)$. Note that

$$
g(t)=e^{\lambda_{0} t} \tilde{g}\left(\frac{1-e^{-\lambda_{0} t}}{2 \lambda_{0}}\right)=e^{\lambda_{0} t} \tilde{g}(s),
$$

which implies

$$
B_{g(t)}(x, \sqrt{t})=B_{e^{\lambda_{0} t} \tilde{g}(s)}(x, \sqrt{t})=B_{\tilde{g}(s)}\left(x, \sqrt{e^{-\lambda_{0} t} t}\right) .
$$

If $t \in\left(0,2 \delta_{0}\right]$, then $s \in\left(0, \frac{4}{3} \delta_{0}\right]$. Note that $\tilde{g}(0)=g(0)$. Therefore, Proposition 3.2 can be applied to obtain the following estimates:

$$
\left\{\begin{aligned}
\left|B_{g(t)}(x, \sqrt{t})\right|_{d \mu_{g(t)}}= & e^{\frac{m \lambda_{0} t}{2}}\left|B_{\tilde{g}(s)}\left(x, \sqrt{e^{-\lambda_{0} t} t}\right)\right|_{d \mu_{\tilde{g}(s)}} \\
& >\frac{1}{2}\left|B_{\tilde{g}(s)}(x, \sqrt{s})\right|_{d \mu_{\tilde{g}(s)}}>\frac{1}{2} \kappa^{\prime} s^{\frac{m}{2}} \triangleq \kappa_{0} t^{\frac{m}{2}}, \\
\sup _{B_{g(t)}(x, \sqrt{t})}|R m|(\cdot, t)= & e^{-\lambda_{0} t} \sup _{B_{\tilde{g}(s)}(x, \sqrt{s})}|\widetilde{R m}|(\cdot, s) \\
& \leq \frac{e^{-\lambda_{0} t}}{100} s^{-1}<\frac{3}{100} e^{-\lambda_{0} t} t^{-1}<t^{-1},
\end{aligned}\right.
$$

for every point $x \in \Omega^{\prime}=B_{g(0)}\left(x_{0}, \frac{3}{4}\right), t \in\left(0,2 \delta_{0}\right]$. So we finish the proof of Theorem 3.1

Remark 3.3. In Proposition 3.2 and Theorem 3.1, one can replace the global Ricci lower bound by a local Ricci lower bound, after improving the statement of Theorem 0.8 of [19], whose proof actually only uses a local Ricci lower bound.

\section{Curvature, Distance and volume estimates}

Under the Ricci flow, evolution of the distance between two points is controlled by the Ricci curvature. By maximum principle, a scalar-flat Ricci flow solution must be Ricci flat. Therefore, the distance between any two points does not depend on the time. In this section, we will develop an "almost" version of this observation. Fix two points in the underlying manifold of a normalized Ricci flow solution. If the normalized scalar curvature is almost zero in the $L^{1}$-sense, then the distance between these two points is almost fixed by the flow. This new estimate is based on Proposition 2.1. Theorem 3.1 and the following estimate of normalized Ricci curvature.

Lemma 4.1. Suppose $\left\{\left(X, x_{0}, g(t)\right),-2 \leq t \leq 1\right\}$ satisfies the following conditions:

- $g(t)$ satisfies the normalized Ricci flow solution

$$
\frac{\partial}{\partial t} g_{i j}=-R_{i j}+\lambda_{0} g_{i j}
$$

where $\lambda_{0}$ is a constant with $\left|\lambda_{0}\right| \leq \frac{1}{100 m^{2}} 3$

\footnotetext{
${ }^{3}$ Note that this is not 1 . In our mind, the flow in this lemma comes from the blowup of a general normalized flow, so the coefficient $\lambda_{0}$ could be very small.
} 
- $|R m|(x, t) \leq \frac{1}{100 m^{2}}$ whenever $x \in B_{g(t)}\left(x_{0}, 100\right), t \in[-2,1]$.

- $\operatorname{inj}\left(x_{0}, t\right) \geq 100$ uniformly for every $t \in[-2,1]$.

Then there exists a large constant $C=C(m)$ such that

$$
\left|R i c-\lambda_{0} g\right|\left(x_{0}, 0\right) \leq C\left\{\int_{-2}^{1} \int_{B_{g(0)}\left(x_{0}, 10\right)}\left|R-m \lambda_{0}\right| d \mu d t\right\}^{\frac{1}{2}} .
$$

Proof. For simplicity of notation, we denote $R i c-\lambda_{0} g$ by $h$ and denote $R-m \lambda_{0}$ by $H$.

Recall that $|h|$ satisfies inequality (44). Locally, $|R m|$ is uniformly bounded. So we should be able to control the $L^{\infty}$-norm of $|h|$ by the $L^{2}$-norm of $|h|$. Actually, define $\Omega=B_{g(0)}\left(x_{0}, 1\right), \Omega^{\prime}=B_{g(0)}\left(x_{0}, \frac{1}{2}\right), D=\Omega \times[-1,0], D^{\prime}=\Omega^{\prime} \times\left[-\frac{1}{2}, 0\right]$. By the second and the third conditions, we obtain that $(\Omega, g(t))$ has a uniform Sobolev constant $\sigma=\sigma(m)$. Similar to Theorem 3.2 of [43], the Moser iteration for the term $h=R i c-\lambda_{0} g$ implies

$$
\sup _{D^{\prime}}|h| \leq C(m)\left\{\iint_{D}|h|^{2} d \mu d t\right\}^{\frac{1}{2}} .
$$

Choose cutoff function $\tilde{\eta}(y, t)=\psi\left(d_{g(t)}\left(y, x_{0}\right)-2\right)$, where $\psi$ is a smooth function which achieves value 1 on $(-\infty, 0]$ and 0 on $[1, \infty)$, which also satisfies $\left|\psi^{\prime}\right| \leq 2$. Recall that $\left|\lambda_{0}\right| \leq \frac{1}{100 \mathrm{~m}^{2}}$. So we have

$$
h(V, V) \leq\left(\frac{m-1}{100 m^{2}}+\left|\lambda_{0}\right|\right) g(V, V) \leq \frac{1}{100 m} g(V, V)
$$

whenever $V \in T X$ and $|R m|(V, V) \leq \frac{1}{100 m^{2}} g(V, V)$. By the evolution of geodesic length, it is easy to check that

$$
\begin{aligned}
& \Omega=B_{g(0)}\left(x_{0}, 1\right) \subset B_{g(t)}\left(x_{0}, 2\right), \\
& B_{g(t)}\left(x_{0}, 3\right) \subset W=B_{g(0)}\left(x_{0}, 10\right),
\end{aligned}
$$

for every $-2 \leq t \leq 1$. Therefore $\tilde{\eta} \equiv 1$ on $\Omega$, and $\tilde{\eta} \equiv 0$ outside $W$ whenever $-2 \leq t \leq 1$.

By the mean value theorem of calculus, we can assume $t_{1}, t_{2}$ satisfies the following properties:

$$
\begin{array}{ll}
-2 \leq t_{1} \leq-1, & \left.\int_{W}|H| d \mu\right|_{t_{1}} \leq \int_{-2}^{-1} \int_{W}|H| d \mu d t \leq \int_{-2}^{1} \int_{W}|H| d \mu d t . \\
0 \leq t_{2} \leq 1, & \left.\int_{W}|H| d \mu\right|_{t_{2}} \leq \int_{0}^{1} \int_{W}|H| d \mu d t \leq \int_{-2}^{1} \int_{W}|H| d \mu d t .
\end{array}
$$


Using the evolution equation of normalized scalar curvature equation (5), similar to the calculation from inequality (37) to inequality (39) of [43, we obtain that

$$
\begin{aligned}
\int_{t_{1}}^{t_{2}} & \int_{\Omega}|h|^{2} d \mu d t \\
& \leq \int_{t_{1}}^{t_{2}} \int_{X} \tilde{\eta}|h|^{2} d \mu d t \\
& =\int_{t_{1}}^{t_{2}} \int_{X} \tilde{\eta}\left(\frac{\partial H}{\partial t}-\frac{1}{2} \Delta H-\lambda_{0} H\right) d \mu d t \\
& =\left.\left(\int_{X} \tilde{\eta} H d \mu\right)\right|_{t_{1}} ^{t_{2}}-\int_{t_{1}}^{t_{2}} \int_{X} H\left(\frac{\partial}{\partial t} \tilde{\eta}+\frac{1}{2} \Delta \tilde{\eta}+\left(\lambda_{0}-\frac{H}{2}\right) \tilde{\eta}\right) d \mu d t \\
& \leq C\left\{\left.\int_{W}|H| d \mu\right|_{t=t_{2}}+\left.\int_{W}|H| d \mu\right|_{t=t_{1}}+\int_{t_{1}}^{t_{2}} \int_{W}|H| d \mu d t\right\} .
\end{aligned}
$$

Note that $[-1,0] \subset\left[t_{1}, t_{2}\right] \subset[-2,1]$. Combining (55), (56), (57), and (58) yields

$$
\sup _{D^{\prime}}|h| \leq C\left\{\iint_{D}|h|^{2} d \mu d t\right\}^{\frac{1}{2}} \leq C\left\{\int_{-2}^{1} \int_{W}|H| d \mu d t\right\}^{\frac{1}{2}},
$$

where $C$ depends only on the dimension $m$.

Combining Lemma 4.1 and Theorem 3.1, we have the following estimate.

Lemma 4.2. Suppose $\left\{\left(X, x_{0}, g(t)\right), 0 \leq t \leq 1\right\}$ satisfies all the conditions in Theorem 3.1. Then

$$
\begin{aligned}
& \left|R i c-\lambda_{0} g\right|(x, s) \leq C(m) s^{-\frac{m+4}{2}}\left\{\int_{0}^{2 s} \int_{\Omega}\left|R-m \lambda_{0}\right| d \mu d t\right\}^{\frac{1}{2}}, \\
& \forall x \in \Omega^{\prime}=B_{g(0)}\left(x_{0}, \frac{1}{2}\right), s \in\left(0, \delta_{0}\right] .
\end{aligned}
$$

Proof. By Theorem 3.1, we have

$$
|R m|(x, t) \leq t^{-1}, \quad\left|B_{g(t)}(x, \sqrt{t})\right|_{d \mu_{g(t)}} \geq \kappa(\sqrt{t})^{m},
$$

for every point $y \in B_{g(0)}\left(x_{0}, \frac{3}{4}\right), t \in\left(0,2 \delta_{0}\right]$.

Fix $x \in \Omega^{\prime}=B_{g(0)}\left(x_{0}, \frac{1}{2}\right), s \in\left(0, \delta_{0}\right]$. By (61), the injectivity radius estimate in 13 yields that

$$
\operatorname{inj}(x, t) \geq \xi \sqrt{s},
$$

for some constant $\xi=\xi(m, \kappa(m))=\xi(m)$ whenever $\frac{s}{2} \leq t \leq 2 s$. Put

$$
A=1000 m \xi^{-1} s^{-\frac{1}{2}}, \quad \tilde{g}(t)=A^{2} g\left(A^{-2} t+s\right) .
$$

Clearly, $\tilde{g}$ satisfies the evolution equation

$$
\frac{\partial}{\partial t} \tilde{g}=-\widetilde{R i c}+A^{-2} \lambda_{0} \tilde{g}
$$


In view of (61) and (62), we have the injectivity radius estimate and curvature estimate required by Lemma 4.1. It follows that

$$
\left|\widetilde{R i c}-A^{-2} \lambda_{0} \tilde{g}\right|^{2}(x, 0) \leq C \int_{-1}^{2} \int_{B_{\tilde{g}_{0}}(x, 10)}\left|\tilde{R}-m A^{-2} \lambda_{0}\right| d \tilde{\mu} d t,
$$

which is the same as the following inequality before scaling:

$$
\left|R i c-\lambda_{0} g\right|^{2}(x, s) \leq C A^{m+4} \int_{s-A^{-2}}^{s+2 A^{-2}} \int_{B_{g(s)}\left(x, 10 A^{-1}\right)}\left|R-m \lambda_{0}\right| d \mu d t .
$$

Recall that in the definition $A=1000 \mathrm{~m}^{-1} \mathrm{~s}^{-\frac{1}{2}}, 1000 \mathrm{~m} \xi^{-1}$ is a constant depending only on $m$. Therefore, (63) implies

$$
\mid \text { Ric }-\lambda_{0} g \mid(x, s) \leq C(m) s^{-\frac{m+4}{2}}\left\{\int_{s-A^{-2}}^{s+2 A^{-2}} \int_{B_{g(s)}\left(x, 10 A^{-1}\right)}\left|R-m \lambda_{0}\right| d \mu d t\right\}^{\frac{1}{2}} .
$$

By inequality (66), whose proof is independent, we obtain that

$$
\begin{aligned}
B_{g(s)}\left(x, 10 A^{-1}\right) & \subset B_{g(s)}\left(x, \frac{1}{8}-C \sqrt{s}\right) \\
& \subset B_{g(0)}\left(x, \frac{1}{8}\right) \subset B_{g(0)}\left(x_{0}, \frac{3}{4}\right) \subset \Omega=B_{g(0)}\left(x_{0}, 1\right) .
\end{aligned}
$$

Then inequality (60) follows from (64), (65) and the fact that $\left[s-A^{-2}, s+2 A^{-2}\right] \subset$ $[0,2 s]$.

Recall Proposition 2.1. estimate (60) implies that distance is almost expanding along the flow.

Lemma 4.3. Suppose $\left\{\left(X, x_{0}, g(t)\right), 0 \leq t \leq 1\right\}$ satisfies all the conditions in Theorem [3.1. Then for every time $t_{0} \in\left(0, \delta_{0}\right]$ and every two points $x_{1}, x_{2} \in \Omega^{\prime}=$ $B_{g(0)}\left(x_{0}, \frac{1}{2}\right)$, we have

$$
d_{g\left(t_{0}\right)}\left(x_{1}, x_{2}\right) \geq d_{g(0)}\left(x_{1}, x_{2}\right)-C \sqrt{t_{0}},
$$

where $C=C(m)$ is a universal constant, $E=\int_{0}^{2 \delta_{0}} \int_{\Omega}\left|R-m \lambda_{0}\right| d \mu d t$. In particular, if $E<\delta_{0}^{m+3}$, then we have

$$
d_{g\left(\delta_{0}\right)}\left(x_{1}, x_{2}\right) \geq d_{g(0)}\left(x_{1}, x_{2}\right)-C E^{\frac{1}{2(m+3)}} .
$$

Proof. Let us first prove inequality (66).

By inequality (7) and inequality (53), we have

$$
\frac{d}{d t} d_{g(t)}\left(x_{1}, x_{2}\right) \geq \frac{1}{2} \lambda_{0} d_{g(0)}\left(x_{1}, x_{2}\right)-C t^{-\frac{1}{2}}, \quad \forall t \in\left(0, t_{0}\right]
$$

where $C$ is a universal constant. In fact, for each time $t$, we see that $|R i c|<$ $(m-1) t^{-1}$ in the set $B_{g(t)}\left(x_{1}, \sqrt{t}\right) \cup B_{g(t)}\left(x_{2}, \sqrt{t}\right)$, in light of (53). Then in (77) 
letting $r_{0}(t)=\sqrt{t}$ we obtain the above inequality. Consequently, we have

$$
\begin{aligned}
& \frac{d}{d t}\left(e^{-\frac{\lambda_{0} t}{2}} d_{g(t)}\left(x_{1}, x_{2}\right)\right) \geq-C e^{-\frac{\lambda_{0} t}{2}} t^{-\frac{1}{2}} \geq-C t^{-\frac{1}{2}}, \\
& \Rightarrow e^{-\frac{\lambda_{0} t_{0}}{2}} d_{g\left(t_{0}\right)}\left(x_{1}, x_{2}\right)-d_{g(0)}\left(x_{1}, x_{2}\right) \geq-C \sqrt{t_{0}}, \\
& \Rightarrow d_{g\left(t_{0}\right)}\left(x_{1}, x_{2}\right) \geq e^{\frac{\lambda_{0} t_{0}}{2}}\left(d_{g(0)}\left(x_{1}, x_{2}\right)-C \sqrt{t_{0}}\right) .
\end{aligned}
$$

If $\lambda_{0} \geq 0$, we have already obtained inequality (66) trivially. If $\lambda_{0}<0$, we have

$$
\begin{aligned}
d_{g\left(t_{0}\right)}\left(x_{1}, x_{2}\right) & \geq\left(d_{g(0)}\left(x_{1}, x_{2}\right)-C \sqrt{t_{0}}\right)+\left(e^{\frac{\lambda_{0} t_{0}}{2}}-1\right)\left(d_{g(0)}\left(x_{1}, x_{2}\right)-C \sqrt{t_{0}}\right) \\
& \geq\left(d_{g(0)}\left(x_{1}, x_{2}\right)-C \sqrt{t_{0}}\right)-C t_{0}\left|d_{g(0)}\left(x_{1}, x_{2}\right)-C \sqrt{t_{0}}\right| \\
& \geq\left(d_{g(0)}\left(x_{1}, x_{2}\right)-C \sqrt{t_{0}}\right)-C t_{0} \\
& \geq d_{g(0)}\left(x_{1}, x_{2}\right)-C \sqrt{t_{0}} .
\end{aligned}
$$

So we finish the proof of inequality (66).

We continue to prove inequality (67). Along the normalized Ricci flow, the derivative of the logarithm of geodesic length is bounded by the term $\left|R i c-\lambda_{0} g\right|$ on the geodesic. Therefore, estimate (60) yields the following inequalities:

$$
\left|\log \frac{d_{g\left(\delta_{0}\right)}\left(x_{1}, x_{2}\right)}{d_{g\left(t_{0}\right)}\left(x_{1}, x_{2}\right)}\right| \leq C \int_{t_{0}}^{\delta_{0}} t^{-\frac{m+4}{2}} E^{\frac{1}{2}} d t \leq C E^{\frac{1}{2}}\left(t_{0}^{-\frac{m+2}{2}}-\delta_{0}^{-\frac{m+2}{2}}\right) \leq C E^{\frac{1}{2}} t_{0}^{-\frac{m+2}{2}} .
$$

It follows that

$$
\begin{aligned}
d_{g\left(\delta_{0}\right)}\left(x_{1}, x_{2}\right) & \geq d_{g\left(t_{0}\right)}\left(x_{1}, x_{2}\right) e^{-C t_{0}^{-\frac{m+2}{2}} E^{\frac{1}{2}}} \\
& \geq\left(d_{g(0)}\left(x_{1}, x_{2}\right)-C t_{0}^{\frac{1}{2}}\right) e^{-C t_{0}^{-\frac{m+2}{2}} E^{\frac{1}{2}}} \\
& =\left(d_{g(0)}\left(x_{1}, x_{2}\right)-C t_{0}^{\frac{1}{2}}\right)+\left(e^{-C t_{0}^{-\frac{m+2}{2}} E^{\frac{1}{2}}}-1\right) \cdot\left(d_{g(0)}\left(x_{1}, x_{2}\right)-C t_{0}^{\frac{1}{2}}\right) \\
& \geq\left(d_{g(0)}\left(x_{1}, x_{2}\right)-C t_{0}^{\frac{1}{2}}\right)-C t_{0}^{-\frac{m+2}{2}} E^{\frac{1}{2}}\left|d_{g(0)}\left(x_{1}, x_{2}\right)-C t_{0}^{\frac{1}{2}}\right| \\
& \geq d_{g(0)}\left(x_{1}, x_{2}\right)-C\left(t_{0}^{\frac{1}{2}}+t_{0}^{-\frac{m+2}{2}} E^{\frac{1}{2}}\right) .
\end{aligned}
$$

If $E<\delta_{0}^{m+3}$, then $E^{\frac{1}{m+3}}<\delta_{0}$. Letting $t_{0}=E^{\frac{1}{m+3}}$ and plugging it into the above inequality, we obtain inequality (67).

Corollary 4.1. Same conditions as in Lemma 4.3. If $E<<\delta_{0}^{m+3}, x_{1} \in \Omega^{\prime}=$ $B_{g(0)}\left(x_{0}, \frac{1}{2}\right)$, then

$$
B_{g\left(\delta_{0}\right)}\left(x_{1}, r-C E^{\frac{1}{2(m+3)}}\right) \subset B_{g(0)}\left(x_{1}, r\right),
$$

for every $0<r<\frac{1}{2}-d_{g(0)}\left(x_{0}, x_{1}\right)$. In particular, we have

$$
B_{g\left(\delta_{0}\right)}\left(x_{0}, r-C E^{\frac{1}{2(m+3)}}\right) \subset B_{g(0)}\left(x_{0}, r\right), \quad \forall 0<r<\frac{1}{2} .
$$

Proof. Direct application of inequality (67). 
Intuitively, an almost expanding map which almost fixes volume must be an almost isometry. This observation can be achieved precisely by Theorem 4.1 . However, in order to obtain Theorem 4.1. we first need an estimate to prevent the distance to expand too fast, which is the meaning of the following Lemma.

Lemma 4.4. Suppose $\left\{\left(X, x_{0}, g(t)\right), 0 \leq t \leq 1\right\}$ satisfies all the conditions in Theorem 3.1 .

Let $\Omega=B_{g(0)}\left(x_{0}, 1\right), \Omega^{\prime}=B_{g(0)}\left(x_{0}, \frac{1}{2}\right)$. For every $l<\frac{1}{2}$, define

$$
\begin{aligned}
& A_{+, l}=\sup _{B_{g(0)}(x, r) \subset \Omega^{\prime}, 0<r \leq l} \omega_{m}^{-1} r^{-m}\left|B_{g(0)}(x, r)\right|_{d \mu_{g(0)}}, \\
& A_{-, l}=\inf _{B_{g\left(\delta_{0}\right)}(x, r) \subset \Omega^{\prime}, 0<r \leq l} \omega_{m}^{-1} r^{-m}\left|B_{g\left(\delta_{0}\right)}(x, r)\right|_{d \mu_{g\left(\delta_{0}\right)}} .
\end{aligned}
$$

If $x_{1}, x_{2} \in \Omega^{\prime \prime}=B_{g(0)}\left(x_{0}, \frac{1}{4}\right), l=d_{g(0)}\left(x_{1}, x_{2}\right)<\frac{1}{8}$, then we have

$$
l-C E^{\frac{1}{2(m+3)}} \leq d_{g\left(\delta_{0}\right)}\left(x_{1}, x_{2}\right) \leq l+C A_{+, 4 l}\left\{\left|\frac{A_{+, l}}{A_{-, l}}-1\right|^{\frac{1}{m}}+l^{-\frac{1}{m}} E^{\frac{1}{2 m(m+3)}}\right\} l
$$

whenever $E=\int_{0}^{2 \delta_{0}} \int_{\Omega}\left|R-m \lambda_{0}\right| d \mu d t<<l^{2(m+3)}$.

Proof. The left hand side of inequality (70) follows directly from inequality (67). So we focus on the proof of the right hand side of inequality (70).

We denote the constant in Lemma 4.3 by $C_{0}$ and fix it in this proof. All the other $C$ 's may be different from line to line.

Among all the geodesic balls in $B_{g(0)}\left(x_{1}, l\right)$, let $B_{g(0)}\left(x, r_{0}\right)$ be the largest geodesic ball (counted by radius under $g(0)$ ) such that

$$
B_{g(0)}\left(x, r_{0}\right) \cap B_{g\left(\delta_{0}\right)}\left(x_{1}, l-C_{0} E^{\frac{1}{2(m+3)}}\right)=\emptyset .
$$

See Figure 3 for intuition.

Claim 4. The radius $r_{0}$ is bounded from above by the following inequality:

$$
r_{0} \leq\left\{\left|\frac{A_{+, l}}{A_{-, l}}-1\right|+C l^{-1} E^{\frac{1}{2(m+3)}}\right\}^{\frac{1}{m}} l+C_{0} E^{\frac{1}{2(m+3)}} .
$$

By definition, $B_{g(0)}\left(x, r_{0}\right)$ and the ball $B_{g\left(\delta_{0}\right)}\left(x_{1}, l-C_{0} E^{\frac{1}{2(m+3)}}\right)$ are disjoint. Moreover, Corollary 4.1 implies that $B_{g(0)}\left(x, r_{0}\right) \cup B_{g\left(\delta_{0}\right)}\left(x_{1}, l-C_{0} E^{\frac{1}{2(m+3)}}\right) \subset$ $B_{g(0)}\left(x_{1}, l\right) \subset \Omega$. Therefore, we have

$$
\left|B_{g(0)}\left(x, r_{0}\right)\right|_{d \mu_{g\left(\delta_{0}\right)}} \leq\left|B_{g(0)}\left(x_{1}, l\right)\right|_{d \mu_{g\left(\delta_{0}\right)}}-\left|B_{g\left(\delta_{0}\right)}\left(x_{1}, l-C_{0} E^{\frac{1}{2(m+3)}}\right)\right|_{d \mu_{g\left(\delta_{0}\right)}} .
$$

In light of the evolution equation of the volume element along the flow, we obtain

$$
\begin{aligned}
& \left|B_{g(0)}\left(x_{1}, l\right)\right|_{d \mu\left(g\left(\delta_{0}\right)\right)}-\left|B_{g(0)}\left(x_{1}, l\right)\right|_{d \mu_{g(0)}} \\
& \quad=\int_{0}^{\delta_{0}} \int_{B_{g(0)}\left(x_{1}, l\right)} \frac{1}{2}\left(m \lambda_{0}-R\right) d \mu_{g(t)} d t \leq \frac{1}{2} \int_{0}^{\delta_{0}} \int_{B_{g(0)}\left(x_{1}, l\right)}\left|m \lambda_{0}-R\right| d \mu_{g(t)} d t \\
& \quad \leq \int_{0}^{2 \delta_{0}} \int_{\Omega}\left|m \lambda_{0}-R\right| d \mu_{g(t)} d t<E .
\end{aligned}
$$




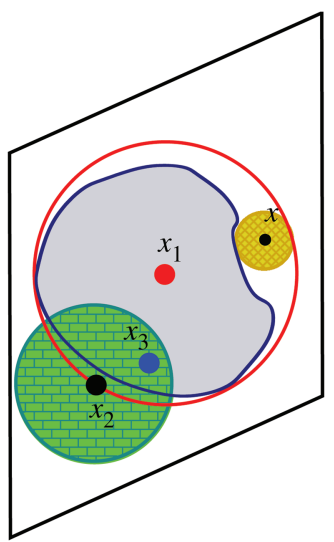

red ball $=B_{g(0)}\left(x_{1}, l\right)$ green ball $=B_{g(0)}\left(x_{2}, 3 r_{0}\right)$

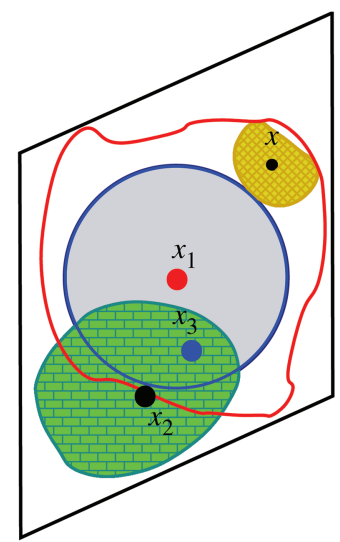

blue ball $=B_{g\left(\delta_{0}\right)}\left(x_{1}, l-C_{0} E^{\frac{1}{2(m+3)}}\right)$ yellow ball $=B_{g(0)}\left(x, r_{0}\right)$

FiguRE 3. The relationship among the balls

Combining the previous two steps yields that

$$
\left|B_{g(0)}\left(x, r_{0}\right)\right|_{d \mu_{g\left(\delta_{0}\right)}} \leq\left|B_{g(0)}\left(x_{1}, l\right)\right|_{d \mu_{g(0)}}-\left|B_{g\left(\delta_{0}\right)}\left(x_{1}, l-C_{0} E^{\frac{1}{2(m+3)}}\right)\right|_{d \mu_{g\left(\delta_{0}\right)}}+E
$$

By Corollary 4.1. we have $B_{g\left(\delta_{0}\right)}\left(x, r_{0}-C_{0} E^{\frac{1}{2(m+3)}}\right) \subset B_{g(0)}\left(x, r_{0}\right)$. Note that $r_{0}<l$ by definition. It follows from the definition of $A_{-, l}$ that

$\left|B_{g(0)}\left(x, r_{0}\right)\right|_{d \mu_{g\left(\delta_{0}\right)}} \geq\left|B_{g\left(\delta_{0}\right)}\left(x, r_{0}-C_{0} E^{\frac{1}{2(m+3)}}\right)\right|_{d \mu_{g\left(\delta_{0}\right)}} \geq A_{-, l}\left(r_{0}-C_{0} E^{\frac{1}{2(m+3)}}\right)^{m}$.

Plugging (73) into (72) yields

$$
\begin{aligned}
A_{-, l} & \left(r_{0}-C_{0} E^{\frac{1}{2(m+3)}}\right)^{m} \\
& \leq\left|B_{g(0)}\left(x_{1}, l\right)\right|_{d \mu_{g(0)}}-\left|B_{g\left(\delta_{0}\right)}\left(x_{1}, l-C_{0} E^{\frac{1}{2(m+3)}}\right)\right|_{d \mu_{g\left(\delta_{0}\right)}}+E \\
& \leq A_{+, l} l^{m}-A_{-, l} l^{m}\left(1-C_{0} l^{-1} E^{\frac{1}{2(m+3)}}\right)^{m}+E \\
& \leq A_{+, l} l^{m}-A_{-, l} l^{m}\left(1-2 m C_{0} l^{-1} E^{\frac{1}{2(m+3)}}\right)+E \\
& \leq l^{m}\left\{\left(A_{+, l}-A_{-, l}\right)+2 m C_{0} A_{-,, l} l^{-1} E^{\frac{1}{2(m+3)}}+l^{-m} E\right\},
\end{aligned}
$$

where we use the fact that $C_{0} l^{-1} E^{\frac{1}{2(m+3)}}<<1$ in the third step and the last step, respectively. By the non-collapsed condition at time $t=\delta_{0}$, we obtain that 
$A_{-, l} \geq C(m, \kappa(m))=c(m)$. By the definition of $A_{-, l}$, we automatically have $A_{-, l} \leq 1$. Note also that $l^{-m} E<<l^{-1} E^{\frac{1}{2(m+3)}}$. We obtain

$$
2 m C_{0} A_{-, l} l^{-1} E^{\frac{1}{2(m+3)}}+l^{-m} E<C A_{-, l} l^{-1} E^{\frac{1}{2(m+3)}} .
$$

Combining (75) and (74) yields

$$
r_{0} \leq\left\{\left|\frac{A_{+, l}}{A_{-, l}}-1\right|+C l^{-1} E^{\frac{1}{2(m+3)}}\right\}^{\frac{1}{m}} l+C_{0} E^{\frac{1}{2(m+3)}} .
$$

Note that there is a point $x_{3} \in B_{g(0)}\left(x_{2}, 3 r_{0}\right)$ such that $x_{3} \in B_{g\left(\delta_{0}\right)}\left(x_{1}, l-\right.$ $C_{0} E^{\frac{1}{2(m+3)}}$ ). Otherwise, let $\alpha$ be a unit speed geodesic (under metric $g(0)$ ) connecting $x_{1}$ and $x_{2}$ such that $\alpha(0)=x_{1}, \alpha(l)=x_{2}$. By triangle inequality, we can see that

$$
\begin{array}{r}
B_{g(0)}\left(\alpha\left(l-\frac{3}{2} r_{0}\right), \frac{5}{4} r_{0}\right) \cap B_{g\left(\delta_{0}\right)}\left(x_{1}, l-C_{0} E^{\frac{1}{2(m+3)}}\right) \\
\subset B_{g(0)}\left(x_{2}, 3 r_{0}\right) \cap B_{g\left(\delta_{0}\right)}\left(x_{1}, l-C_{0} E^{\frac{1}{2(m+3)}}\right)=\emptyset,
\end{array}
$$

which contradicts to the definition of $r_{0}$.

Claim 5. There exists a constant $C=C(m)$ such that

$$
d_{g\left(\delta_{0}\right)}\left(x_{2}, x_{3}\right) \leq C A_{+, 4 l} \max \left\{3 C_{0} E^{\frac{1}{2(m+3)}}, r_{0}\right\} .
$$

We first consider the case that $r_{0}>3 C_{0} E^{\frac{1}{2(m+3)}}$.

Under metric $g(0)$, let $\gamma$ be the shortest geodesic connecting $x_{2}, x_{3}$. Clearly, $|\gamma|_{g(0)} \leq 3 r_{0}$. Under the metric $g\left(\delta_{0}\right), \gamma$ may not be the shortest geodesic. However, it is still a smooth curve. Cover the curve $\gamma$ by geodesic balls $B_{g\left(\delta_{0}\right)}\left(z_{i}, r_{0}\right)$ with the following properties:

- $z_{i} \in \gamma, \quad \forall i \in\{1, \ldots, N\}$

- $\gamma \subset \bigcup_{i=1}^{N} B_{g\left(\delta_{0}\right)}\left(z_{i}, r_{0}\right)$;

- $B_{g\left(\delta_{0}\right)}\left(z_{i}, \frac{r_{0}}{2}\right)$ are disjoint.

Since $z_{i} \in \gamma$, we have $z_{i} \in B_{g(0)}\left(x_{2}, 3 r_{0}\right) \subset B_{g(0)}\left(x_{0}, \frac{1}{2}\right)$. Note that $r_{0}>3 C_{0} E^{\frac{1}{2(m+3)}}$, and Corollary 4.1 implies that

$$
\begin{gathered}
B_{g\left(\delta_{0}\right)}\left(z_{i}, \frac{r_{0}}{2}\right) \subset B_{g(0)}\left(z_{i}, \frac{r_{0}}{2}+C_{0} E^{\frac{1}{2(m+3)}}\right) \subset B_{g(0)}\left(z_{i}, r_{0}\right) \subset B_{g(0)}\left(x_{2}, 4 r_{0}\right) \\
\subset B_{g(0)}\left(x_{0}, \frac{1}{4}+4 r_{0}\right) \subset \Omega^{\prime}=B_{g(0)}\left(x_{0}, \frac{1}{2}\right) \subset \Omega=B_{g(0)}\left(x_{0}, 1\right) .
\end{gathered}
$$

Note that $B_{g\left(\delta_{0}\right)}\left(z_{i}, \frac{r_{0}}{2}\right)$ are disjoint, and we obtain

$$
\sum_{i=1}^{N}\left|B_{g\left(\delta_{0}\right)}\left(z_{i}, \frac{r_{0}}{2}\right)\right|_{d \mu_{g(0)}} \leq\left|B_{g(0)}\left(x_{2}, 4 r_{0}\right)\right|_{d \mu_{g(0)}} .
$$

By the evolution equation of volume form and (78), we have

$$
\left.\left|\sum_{i=1}^{N}\right| B_{g\left(\delta_{0}\right)}\left(z_{i}, \frac{r_{0}}{2}\right)\right|_{d \mu_{g(0)}}-\sum_{i=1}^{N}\left|B_{g\left(\delta_{0}\right)}\left(z_{i}, \frac{r_{0}}{2}\right)\right|_{d \mu_{g\left(\delta_{0}\right)}} \mid<E .
$$


It follows from (79) and (80) that

$$
\sum_{i=1}^{N}\left|B_{g\left(\delta_{0}\right)}\left(z_{i}, \frac{r_{0}}{2}\right)\right|_{d \mu_{g\left(\delta_{0}\right)}} \leq\left|B_{g(0)}\left(x_{2}, 4 r_{0}\right)\right|_{d \mu_{g(0)}}+E .
$$

Since $r_{0}<l$, the definition of $A_{-, l}, A_{+, l}$ implies the following inequalities:

$$
\begin{aligned}
& \left|B_{g\left(\delta_{0}\right)}\left(z_{i}, \frac{r_{0}}{2}\right)\right|_{d \mu_{g\left(\delta_{0}\right)}} \geq A_{-, l}\left(\frac{r_{0}}{2}\right)^{m}, \quad \forall i \in\{1, \ldots, N\} ; \\
& \left|B_{g(0)}\left(x_{1}, 4 r_{0}\right)\right|_{d \mu_{g(0)}} \leq A_{+, 4 l}\left(4 r_{0}\right)^{m} .
\end{aligned}
$$

Combining (81), (82), and (83), we obtain

$$
\frac{N A_{-, l}}{2^{m}} r_{0}^{m} \leq 4^{m} A_{+, 4 l} r_{0}^{m}+E, \Rightarrow N \leq 2^{m}\left(4^{m} A_{+, 4 l}+E r_{0}^{-m}\right) A_{-, l}^{-1} .
$$

Recall that $\bigcup_{i=1}^{N} B_{g_{i}\left(\delta_{0}\right)}\left(z_{i}, r_{0}\right)$ is a covering of $\gamma$. Therefore, (84) implies

$$
d_{g\left(\delta_{0}\right)}\left(x_{2}, x_{3}\right) \leq 2 N r_{0} \leq 2^{m+1}\left(4^{m} A_{+, 4 l}+E r_{0}^{-m}\right) A_{-, l}^{-1} r_{0} .
$$

On one hand, by (52), the non-collapsed condition at time $t=\delta_{0}$ implies that $A_{-, l}^{-1}$ is bounded from above uniformly. On the other hand, $A_{+, 4 l}$ is bounded from below in view of the volume comparison and (51). Therefore, the fact that $r_{0}>3 C_{0} E^{\frac{1}{2(m+3)}}$ implies $\operatorname{Er}_{0}^{-m}<\left(3 C_{0}\right)^{-m} E^{\frac{m+6}{2(m+3)}}<C A_{+, 4 l}$. Consequently, we can simplify (85) to

$$
d_{g\left(\delta_{0}\right)}\left(x_{2}, x_{3}\right) \leq C A_{+, 4 l} r_{0},
$$

which is the same as (77) under our assumption $r>3 C_{0} E^{\frac{1}{2(m+3)}}$. If $r_{0} \leq 3 C_{0} E^{\frac{1}{2(m+3)}}$, we can repeat the previous argument by choosing covering balls of radius $3 C_{0} E^{\frac{1}{2(m+3)}}$. The details are similar, so we omit them.

Now we can combine Claims 4 and 5 to obtain the precise upper bound of $d_{g\left(\delta_{0}\right)}\left(x_{2}, x_{3}\right)$. If $r_{0} \leq 3 C_{0} E^{\frac{1}{2(m+3)}}$, we obtain

$$
d_{g\left(\delta_{0}\right)}\left(x_{2}, x_{3}\right) \leq C A_{+, 4 l} E^{\frac{1}{2(m+3)}}<C A_{+, 4 l} l^{\frac{m-1}{m}} E^{\frac{1}{2 m(m+3)}}
$$

since $E<<l^{2(m+3)}$. If $r_{0}>3 C_{0} E^{\frac{1}{2(m+3)}}$, then we have

$$
\begin{aligned}
d_{g\left(\delta_{0}\right)}\left(x_{2}, x_{3}\right) & \leq C A_{+, 4 l}\left\{\left\{\left|\frac{A_{+, l}}{A_{-, l}}-1\right|+C l^{-1} E^{\frac{1}{2(m+3)}}\right\}^{\frac{1}{m}} l+C_{0} E^{\frac{1}{2(m+3)}}\right\} \\
& \leq C A_{+, 4 l}\left\{\left|\frac{A_{+, l}}{A_{-, l}}-1\right|^{\frac{1}{m}} l+C^{\frac{1}{m}} l^{\frac{m-1}{m}} E^{\frac{1}{2 m(m+3)}}+C_{0} E^{\frac{1}{2(m+3)}}\right\} \\
& \leq C A_{+, 4 l}\left\{\left|\frac{A_{+, l}}{A_{-, l}}-1\right|^{\frac{1}{m}}+l^{-\frac{1}{m}} E^{\frac{1}{2 m(m+3)}}\right\} l .
\end{aligned}
$$

Therefore, triangle inequality yields that

$$
\begin{aligned}
d_{g\left(\delta_{0}\right)}\left(x_{1}, x_{2}\right) & \leq d_{g\left(\delta_{0}\right)}\left(x_{1}, x_{3}\right)+d_{g\left(\delta_{0}\right)}\left(x_{3}, x_{2}\right) \leq l-C_{0} E^{\frac{1}{2(m+3)}}+d_{g\left(\delta_{0}\right)}\left(x_{3}, x_{2}\right) \\
& <l+C A_{+, 4 l}\left\{\left|\frac{A_{+, l}}{A_{-, l}}-1\right|^{\frac{1}{m}}+l^{-\frac{1}{m}} E^{\frac{1}{2 m(m+3)}}\right\} l .
\end{aligned}
$$


By refining the estimate in Lemma 4.4, we are able to prove that the distance is almost fixed whenever the normalized scalar curvature is almost zero.

Theorem 4.1. Suppose $\left\{\left(X, x_{0}, g(t)\right), 0 \leq t \leq 1\right\}$ satisfies all the conditions in Theorem 3.1 . Then for every two points $x_{1}, x_{2} \in \Omega^{\prime \prime}=B_{g(0)}\left(x_{0}, \frac{1}{4}\right), l=d_{g(0)}\left(x_{1}, x_{2}\right)$, we have

$$
l-C E^{\frac{1}{2(m+3)}} \leq d_{g\left(\delta_{0}\right)}\left(x_{1}, x_{2}\right) \leq l+C l E^{\frac{1}{3 m(m+3)}}
$$

whenever $E=\int_{0}^{2 \delta_{0}} \int_{\Omega}\left|R-m \lambda_{0}\right| d \mu d t<<l^{6(m+3)}$. Here $C=C\left(m, \delta_{0}(m)\right)=C(m)$.

Proof. The first inequality of (89) is the same as the one in (70). So we only need to show the second inequality of (89).

At time $t=\delta_{0},|R m|$ is uniformly bounded, injectivity radius is uniformly bounded from below. Therefore, the Rauch comparison theorem can be applied to obtain a lower bound of $A_{-, r}$. At time $t=0$, Ricci curvature is bounded from below. So the Bishop volume comparison theorem implies an upper bound of $A_{+, r}$. In short, we have

$$
A_{+, r} \leq 1+C r^{2}, \quad A_{-, r} \geq 1-C r^{2},
$$

whenever $r<\xi=\xi\left(m, \kappa(m), \delta_{0}(m)\right)=\xi(m)$. It follows that

$$
C A_{+, 4 r}\left\{\left|\frac{A_{+, r}}{A_{-, r}}-1\right|^{\frac{1}{m}}+r^{-\frac{1}{m}} E^{\frac{1}{2 m(m+3)}}\right\} \leq C\left\{r^{\frac{2}{m}}+r^{-\frac{1}{m}} E^{\frac{1}{2 m(m+3)}}\right\} .
$$

By (70) and (90), we have

$$
d_{g\left(\delta_{0}\right)}\left(y_{1}, y_{2}\right) r^{-1} \leq 1+C\left\{r^{\frac{2}{m}}+r^{-\frac{1}{m}} E^{\frac{1}{2 m(m+3)}}\right\},
$$

whenever $y_{1}, y_{2} \in B_{g(0)}\left(x_{0}, \frac{1}{4}\right)$ and $d_{g(0)}\left(y_{1}, y_{2}\right)=r<\xi$.

Fix a big integer number $N>\xi^{-1} l$. Let $\gamma$ be a unit speed shortest geodesic connecting $x_{1}, x_{2}$ such that $\gamma(0)=x_{1}, \gamma(l)=x_{2}$. Define $z_{i}=\gamma\left(N^{-1} i l\right)$. Clearly, $z_{0}=x_{1}, z_{N}=x_{2}$. Since $d_{g\left(\delta_{0}\right)}\left(z_{i}, z_{i+1}\right)=N^{-1} l<\xi$ for every $i=0, \ldots, N-1$, it follows from (91) that

$$
\frac{d_{g\left(\delta_{0}\right)}\left(z_{i}, z_{i+1}\right)}{N^{-1} l} \leq 1+C\left\{N^{-\frac{2}{m}} l^{\frac{2}{m}}+N^{\frac{1}{m}} l^{-\frac{1}{m}} E^{\frac{1}{2 m(m+3)}}\right\} .
$$

In view of triangle inequality, we obtain

$$
\begin{aligned}
\frac{d_{g\left(\delta_{0}\right)}\left(x_{1}, x_{2}\right)}{N^{-1} l} & \leq \frac{\sum_{i=0}^{N} d_{g\left(\delta_{0}\right)}\left(z_{i}, z_{i+1}\right)}{N^{-1} l} \\
& \leq N\left\{1+C\left\{N^{-\frac{2}{m}} l^{\frac{2}{m}}+N^{\frac{1}{m}} l^{-\frac{1}{m}} E^{\frac{1}{2 m(m+3)}}\right\}\right\},
\end{aligned}
$$

which in turn implies that

$$
d_{g\left(\delta_{0}\right)}\left(x_{1}, x_{2}\right) l^{-1} \leq l+C\left\{N^{-\frac{2}{m}} l^{\frac{2}{m}}+N^{\frac{1}{m}} l^{-\frac{1}{m}} E^{\frac{1}{2 m(m+3)}}\right\} .
$$

Let $N \sim l E^{-\frac{1}{6(m+3)}}>l \xi^{-1}$. Then (92) yields that $d_{g\left(\delta_{0}\right)}\left(x_{1}, x_{2}\right) l^{-1} \leq 1+C E^{\frac{1}{3 m(m+3)}}$.

Based on Theorem 4.1] we are ready to prove a gap theorem. 
Theorem 4.2 (Gap theorem). There exists a big constant $L_{0}=L_{0}(m)$ such that the following properties hold.

Suppose $\left\{\left(X, x_{0}, g(t)\right), 0 \leq t \leq 1\right\}$ satisfies the same conditions as in Theorem 3.1 . Then for every $0<r<\frac{1}{4}$, we have

$$
r^{-1} d_{G H}\left(\left(B_{g(0)}\left(x_{0}, r\right), g(0)\right),\left(B_{g\left(\delta_{0}\right)}\left(x_{0}, r\right), g\left(\delta_{0}\right)\right)\right)<L_{0} r^{-1} E^{\frac{1}{3 m(m+3)}},
$$

whenever $E=\int_{0}^{2 \delta_{0}} \int_{B_{g(0)}\left(x_{0}, 1\right)}\left|R-m \lambda_{0}\right| d \mu d t<<r^{6(m+3)}$. Moreover, we have

$$
r^{-1} d_{G H}\left(\left(B_{g(0)}\left(x_{0}, r\right), g(0)\right),\left(B(0, r), g_{\mathbb{E}}\right)\right)<L_{0} r^{2},
$$

whenever $E<<r^{9 m(m+3)}, r<<1$. Here $B(0, r)$ is the ball with radius $r$ in the Euclidean space $\mathbb{R}^{m}$.

Proof. By (89), we have

$$
\left|d_{g(0)}\left(x_{1}, x_{2}\right)-d_{g\left(\delta_{0}\right)}\left(x_{1}, x_{2}\right)\right|<C \max \left\{E^{\frac{1}{3 m(m+3)}}, E^{\frac{1}{2(m+3)}}\right\}<C E^{\frac{1}{3 m(m+3)}}
$$

for every two points $x_{1}, x_{2} \in B_{g(0)}\left(x_{0}, \frac{1}{4}\right)$ satisfying $d_{g(0)}\left(x_{1}, x_{2}\right)>>E^{\frac{1}{6(m+3)}}$. In particular, if $d_{g(0)}\left(x_{1}, x_{2}\right)$ is comparable with $E^{\frac{1}{3 m(m+3)}}>>E^{\frac{1}{6(m+3)}}$, then (95) holds. This means that the identity map is a $C E^{\frac{1}{3 m(m+3)}}$-approximation map from $\left(B_{g(0)}\left(x_{0}, r\right), g(0)\right)$ to $\left(B_{g(0)}\left(x_{0}, r\right), g\left(\delta_{0}\right)\right)$. Therefore, we have

$$
d_{G H}\left(\left(B_{g(0)}\left(x_{0}, r\right), g(0)\right),\left(B_{g(0)}\left(x_{0}, r\right), g\left(\delta_{0}\right)\right)\right)<C E^{\frac{1}{3 m(m+3)}} .
$$

On the other hand, (89) implies that

$$
B_{g\left(\delta_{0}\right)}\left(x_{0}, r-C E^{\frac{1}{2(m+3)}}\right) \subset B_{g(0)}\left(x_{0}, r\right) \subset B_{g\left(\delta_{0}\right)}\left(x_{0}, r+C E^{\frac{1}{3 m(m+3)}}\right),
$$

which in turn yields that

$$
d_{G H}\left(\left(B_{g(0)}\left(x_{0}, r\right), g\left(\delta_{0}\right)\right),\left(B_{g\left(\delta_{0}\right)}\left(x_{0}, r\right), g\left(\delta_{0}\right)\right)\right)<C E^{\frac{1}{3 m(m+3)}}
$$

by the definition of the Gromov-Hausdorff distance. Combining (96) and (97), we obtain

$$
d_{G H}\left(\left(B_{g(0)}\left(x_{0}, r\right), g(0)\right),\left(B_{g\left(\delta_{0}\right)}\left(x_{0}, r\right), g\left(\delta_{0}\right)\right)\right)<C E^{\frac{1}{3 m(m+3)}},
$$

whose scaling-invariant form on the left hand side is (93).

At time $t=\delta_{0}$, around $x_{0},|R m|$ is uniformly bounded, and the injectivity radius is uniformly bounded from below. Using the exponential map, one can construct an approximation map from the Euclidean ball to the geodesic ball. It is not hard to see that

$$
r^{-1} d_{G H}\left(\left(B_{g\left(\delta_{0}\right)}\left(x_{0}, r\right), g\left(\delta_{0}\right)\right),\left(B(0, r), g_{\mathbb{E}}\right)\right)<C r^{2}
$$

whenever $r$ is very small. It follows from (93) and (98) that

$$
r^{-1} d_{G H}\left(\left(B_{g(0)}\left(x_{0}, r\right), g(0)\right),\left(B(0, r), g_{\mathbb{E}}\right)\right)<C\left\{r^{2}+r^{-1} E^{\frac{1}{3 m(m+3)}}\right\}<C r^{2}
$$

whenever $E<r^{9 m(m+3)}$. Therefore, (94) is proved. Letting $L_{0}$ be the maximum of all the $C$ 's that appear in this proof, we obtain Theorem 4.2 . 


\section{Structure of Limit SPACE}

This section is devoted to prove the structure theorems, Theorems 11 and 2 , respectively.

5.1. Riemannian case. Suppose $\left(X_{i}, x_{i}, g_{i}\right)$ is a sequence of almost Einstein manifolds. Let $(\bar{X}, \bar{x}, \bar{g})$ be the limit space of $\left(X_{i}, x_{i}, g_{i}\right)$, and $\bar{\lambda}$ be the limit of $\lambda_{i}$. In this section, we shall use the estimates developed in previous sections to show the structure of $\bar{X}$.

A tangent space $(\hat{Y}, \hat{y}, \hat{g})$ at a point $y \in \bar{X}$ is the pointed-Gromov-Hausdorff limit of $\left(\bar{X}, y, \epsilon_{j}^{-2} \bar{g}\right)$ for some sequence $\epsilon_{j} \rightarrow 0$. A point $y \in \bar{X}$ is called regular if every tangent cone at $y$ is isometric to the Euclidean space $\left(\mathbb{R}^{m}, 0, g_{\mathbb{E}}\right)$. A point $y \in X$ is called singular if it is not regular; i.e., at $y$, there exists a tangent space $(\hat{Y}, \hat{y}, \hat{g})$ which is not isometric to the Euclidean space. By the fundamental work in [6], one sees that every tangent space is a metric cone. Moreover, a tangent cone is Gromov-Hausdorff close to the Euclidean space if and only if the volume of the standard unit ball in the tangent cone is close to $\omega_{m}$, the volume of the unit ball in $\mathbb{R}^{m}$. Under the non-collapsed and Ricci lower bound condition, the Hausdorff measure converges whenever the Gromov-Hausdorff convergence happens. This inspires us to define the function $\mathcal{U}$ on $\bar{X} \times(0, \infty)$ as follows. For every point $y \in \bar{X}$, define $\mathcal{U}(y, r) \triangleq \omega_{m}^{-1} r^{-m}|B(y, r)|$. Since the space $\bar{X}$ inherits the Bishop-Gromov volume comparison property from the limit process 4 we see that $\lim _{r \rightarrow 0} \mathcal{U}(y, r)$ is a well-defined positive number, which we denote by $\mathcal{U}(y)$. Clearly, a point $y$ is singular if and only if $\mathcal{U}(y)<1$. However, by using the special property of almost Einstein limit, this property can be improved.

Proposition 5.1. $y \in \bar{X}$ is a singular point if and only if $\mathcal{U}(y) \leq\left(1-\frac{\delta_{0}}{2}\right)$.

Proof. It suffices to show that $y$ is regular whenever $\mathcal{U}(y)>\left(1-\frac{\delta_{0}}{2}\right)$.

Suppose $\mathcal{U}(y)>\left(1-\frac{\delta_{0}}{2}\right)$. By definition of $\mathcal{U}(y)$, there exists a sequence of $\rho_{j} \rightarrow 0$ such that

$$
\omega_{m}^{-1} \rho_{j}^{-m}\left|B\left(y, \rho_{j}\right)\right|>\left(1-\frac{1}{2} \delta_{0}\right) .
$$

Denote the pointed-Gromov-Hausdorff limit of $\left(\bar{X}, y, \rho_{j}^{-2} \bar{g}\right)$ by $(\hat{Y}, \hat{y}, \hat{g})$, which is a tangent cone of $\bar{X}$ at the point $y$. By a careful choice of diagonal subsequence if necessary, we can assume $(\hat{Y}, \hat{y}, \hat{g})$ as the pointed-Gromov-Hausdorff limit of $\left(X_{i_{j}}, y_{i_{j}}, \rho_{j}^{-2} g_{i_{j}}\right)$, which is a new sequence of almost Einstein manifolds. For brevity, we drop some subindexes and look at $(\hat{Y}, \hat{y}, \hat{g})$ as the almost Einstein limit of $\left(X_{j}, y_{j}, h_{j}\right)$, where $h_{j}=\rho_{j}^{-2} g_{i_{j}}$. By volume continuity, we have

$$
\rho_{j}^{-m}\left|B\left(y, \rho_{j}\right)\right|>\omega_{m}\left(1-\frac{1}{2} \delta_{0}\right), \Rightarrow\left|B\left(y_{j}, 1\right)\right|_{d \mu_{h_{j}}}>\left(1-\delta_{0}\right) \omega_{m} .
$$

\footnotetext{
${ }^{4}$ We use the volume convergence theorem of 19 here again. This theorem plays a fundamental role in our approach.
} 
Clearly, $\operatorname{Ric}_{h_{j}} \geq-(m-1) \rho_{j}^{2}$ on $X_{j}$. Therefore, Theorem 4.2 applies. Fix an arbitrary small $r>0$, by inequality (94), we see that

$$
\begin{aligned}
& r^{-1} d_{G H}\left(\left(B_{h_{j}}\left(y_{j}, r\right), h_{j}\right),\left(B(0, r), g_{\mathbb{E}}\right)\right)<C r^{2} \\
& \quad \Rightarrow r^{-1} d_{G H}\left(\left(B_{\hat{g}}(\hat{y}, r), \hat{g}\right),\left(B(0, r), g_{\mathbb{E}}\right)\right) \leq C r^{2} .
\end{aligned}
$$

Consequently, every tangent space of $\hat{Y}$ at $\hat{y}$ is the Euclidean space $\mathbb{R}^{m}$. On the other hand, we already know $\hat{Y}$ is a metric cone with vertex $\hat{y}$. These two conditions force that $\hat{Y}$ is isometric to $\mathbb{R}^{m}$. Henceforth, $y$ is a regular point.

By some routine argument, the following Corollary is obvious now.

Corollary 5.1. There exists a constant $\bar{\epsilon}=\bar{\epsilon}(m)>0$ with the following property.

Suppose $y \in \bar{X},(\hat{Y}, \hat{y}, \hat{g})$ is a tangent space of $\bar{X}$ at $y$, and $B(0,1)$ is the unit ball in the Euclidean space $\mathbb{R}^{m}$. Then $\hat{Y}$ is isometric to $\mathbb{R}^{m}$ if and only if

$$
d_{G H}\left(\left(B_{\hat{g}}(\hat{y}, 1), \hat{g}\right),\left(B(0,1), g_{\mathbb{E}}\right)\right)<\bar{\epsilon} .
$$

Using the notation of [6], Corollary 5.1 implies $\mathcal{R}=\mathcal{R}_{\bar{\epsilon}}$. Therefore, we have separated the singular points from the regular points substantially. Then by using regularity results from the Ricci flow, we can smoothen the regular part $\mathcal{R}$.

Proposition 5.2. Suppose $y \in \bar{X}$ is a regular point. Then there exists a constant $r=r(y)$ with the following properties:

- $\left(B_{\bar{g}}(y, r), \bar{g}\right)$ is geodesic convex; i.e., every shortest geodesic connecting two points in $B_{\bar{g}}(y, r)$ cannot escape it.

- There exist a region $D \subset \mathbb{R}^{m}$ and a smooth metric tensor $g_{D}$ on $D$ such that $\left(B_{\bar{g}}(y, r), \bar{g}\right)$ is isometric to $\left(D, g_{D}\right)$.

- $\operatorname{Ric}_{\bar{g}}(y)-\bar{\lambda} \bar{g}(y)=0$.

Proof. Since $y$ is regular, $\mathcal{U}(y)=1$. So we can find $r_{0}=r_{0}(y)$ such that $\mathcal{U}(y, \rho)>$ $\left(1-\frac{\delta_{0}}{2}\right)$ for every $0<\rho<r_{0}(y)$. Suppose $y_{i} \rightarrow y$ as $\left(X_{i}, x_{i}, g_{i}\right)$ converges to $(\bar{X}, \bar{x}, \bar{g})$. By volume continuity, we have for large $i$

$$
r_{0}^{-m}\left|B_{g_{i}}\left(y_{i}, r_{0}\right)\right|_{d \mu_{g_{i}}}>\left(1-\delta_{0}\right) \omega_{m} .
$$

Without loss of generality, we choose $r_{0}<\sqrt{\delta_{0}}$. Let $\tilde{g}_{i}=r_{0}^{-2} g_{i}, \Omega_{i}=B_{\tilde{g}_{i}}\left(y_{i}, 1\right)$. Then we have

$$
\operatorname{Ric}_{\tilde{g}_{i}}(x) \geq-(m-1) r_{0}^{2}>-(m-1) \delta_{0}, \forall x \in \Omega_{i} ; \quad\left|B_{\tilde{g}_{i}}\left(y_{i}, 1\right)\right|_{d \mu_{\tilde{g}_{i}}} \geq\left(1-\delta_{0}\right) \omega_{m} .
$$

So we can apply Theorem 4.2 for the new almost Einstein sequence $\left(X_{i}, y_{i}, \tilde{g}_{i}\right)$. By (93), it turns out that

$$
\lim _{i \rightarrow \infty} 8 d_{G H}\left(\left(B_{\tilde{g}_{i}(0)}\left(y_{i}, \frac{1}{8}\right), \tilde{g}_{i}(0)\right),\left(B_{\tilde{g}_{i}\left(\delta_{0}\right)}\left(y_{i}, \frac{1}{8}\right), \tilde{g}_{i}\left(\delta_{0}\right)\right)\right)=0 .
$$

Denote the common Gromov-Hausdorff limit of the two sequences of geodesic balls in (101) by $\left(B_{\tilde{g}_{\infty}}\left(y_{\infty}, \frac{1}{8}\right), \tilde{g}_{\infty}\right)$. Note that $B_{\tilde{g}_{i}\left(\delta_{0}\right)}\left(y_{i}, \frac{1}{8}\right) \subset B_{\tilde{g}_{i}(0)}\left(y_{i}, \frac{1}{2}\right)$ by Theorem 4.1. Therefore, Theorem 3.1 and Shi's local estimate (cf. 34]) imply that there 
exist a small positive number $\rho_{0}<<\min \left\{\frac{1}{8}, \delta_{0}\right\}$ and large positive constants $C_{k}$ such that

$$
\begin{aligned}
& \quad \inf _{B_{\tilde{g}_{i}\left(\delta_{0}\right)}\left(y_{i}, \rho_{0}\right)} i n j_{\tilde{g}_{i}\left(\delta_{0}\right)}(x)>>\rho_{0} ; \\
& \quad \sup _{B_{\tilde{g}_{i}\left(\delta_{0}\right)}\left(y_{i}, \rho_{0}\right)}\left|\nabla^{k} R m\right|_{\tilde{g}_{i}\left(\delta_{0}\right)}(x)<<C_{k} \rho_{0}^{-2-k}, \forall k \in \mathbb{Z}^{+} \cup\{0\} .
\end{aligned}
$$

Consequently, $\left(B_{\tilde{g}_{\infty}}\left(y_{\infty}, \rho_{0}\right), \tilde{g}_{\infty}\right)$ is a convex smooth geodesic ball. Denote $h=$ $r_{0}^{2} \tilde{g}_{\infty}$. Of course, $\left(B_{h}\left(y_{\infty}, r_{0} \rho_{0}\right), h\right)$ is a convex smooth geodesic ball. By exponential map with respect to $h$, we can find $D \subset \mathbb{R}^{m}$ and smooth $g_{D}$ such that $\left(D, g_{D}\right)$ is isometric to $\left(B_{h}\left(y_{\infty}, r_{0} \rho_{0}\right), h\right)$, which is the Gromov-Hausdorff limit of $\left(B\left(y_{i}, r_{0} \rho_{0}\right), g_{i}(0)\right)$. So we finish the proof of the first two properties by letting $r=r_{0} \rho_{0}$. The last property follows from Lemma 4.2. Actually, (100) guarantees that we can apply inequality (60) to obtain

$$
\begin{aligned}
\left|R i c_{g_{i}}-\lambda_{i} g_{i}\right|\left(y_{i}, \delta_{0} r_{0}^{2}\right) & =r_{0}^{-2}\left|R i c_{\tilde{g}_{i}}-\lambda_{i} r_{0}^{2} \tilde{g}_{i}\right|\left(y_{i}, \delta_{0}\right) \\
& <C\left\{\int_{0}^{2 \delta_{0}} \int_{X_{i}}\left|R-m r_{0}^{2} \lambda_{i}\right|_{\tilde{g}_{i}(t)} d \mu_{\tilde{g}_{i}(t)} d t\right\}^{\frac{1}{2}} \rightarrow 0,
\end{aligned}
$$

where $C=C\left(m, r_{0}, \delta_{0}\right)$. Since $\bar{g}$ is the smooth limit of $g_{i}\left(\delta_{0} r_{0}^{2}\right)$ around $y$, we obtain $\operatorname{Ric}_{\bar{g}}(y)=\bar{\lambda} \bar{g}(y)$.

For brevity, for every point $x \in \bar{X}$, define the volume radius

$$
r_{V}(x) \triangleq \max \left\{r>0 \mid \mathcal{U}(x, r) \geq\left(1-\frac{1}{2} \delta_{0}\right)\right\}
$$

whenever the set is nonempty. Otherwise, let $r_{V}(x)=0$. Define $\mathcal{V}_{r} \triangleq\left\{x \in \bar{X} \mid r_{V}(x)\right.$ $\leq r\}$, the set of points whose volume radius is not greater than $r$. Clearly, $\mathcal{V}_{0}$ is nothing but the singular set $\mathcal{S}$. Using the notation in [6], for a metric space $Z$, we assume $z^{*}$ is the vertex of the metric cone $C(Z)$. Then for every pair of small positive constants $\eta, \xi$ and radius $0<r<\xi$, we define

$\mathcal{S}_{\eta,(r, \xi)}^{k}=\left\{y \in \bar{X} \mid \inf _{r<s<\xi} s^{-1} d_{G H}\left(B(y, s), B\left(\left(\underline{0}, z^{*}\right), s\right)\right) \geq \eta\right.$, for all $\left.\mathbb{R}^{k+1} \times C(Z)\right\}$.

Note that our $\mathcal{S}_{\eta,(r, 1)}^{k}$ is $\mathcal{S}_{\eta, r}^{k}$ in [12]. By Theorem 1.10 of [12, a standard rescaling argument shows that for every $\xi<1$ and $\eta<<1$,

$$
\xi^{-m}\left|B(y, 2 \xi) \cap \mathcal{S}_{\eta,(r, \xi)}^{m-2}\right| \leq C(m, \kappa, \eta)\left(\frac{r}{\xi}\right)^{2-\eta}
$$

whenever $y \in B(\bar{x}, 2)$. Consequently, the non-collapsed condition and a ballcovering argument imply that

$$
\left|B(\bar{x}, 2) \cap \mathcal{S}_{\eta,(r, \xi)}^{m-2}\right| \leq C(m, \kappa, \eta) \xi^{-2+\eta} r^{2-\eta} .
$$

In particular, we have

$$
\left|B(\bar{x}, 2) \cap \mathcal{S}_{\eta,(r, \eta)}^{m-2}\right|<C(m, \kappa, \eta) r^{2-\eta} .
$$

Therefore, we can obtain

$$
\left|B(\bar{x}, 2) \cap \mathcal{V}_{r}\right| \leq C(m, \kappa, \eta) r^{2-\eta}
$$


if we can prove $\mathcal{V}_{r} \subset \mathcal{S}_{\eta,(r, \eta)}^{m-2}$. In fact, this relationship follows from the following Lemma.

Lemma 5.1. There exists a constant $\eta_{0}=\eta_{0}(m, \kappa)$ with the following property.

Suppose that $(Y, g)$ is an m-dimensional complete Riemannian manifold, Ric $(x)$ $\geq-(m-1)$ in a geodesic ball $B\left(y_{0}, 2\right),\left|B\left(y_{0}, 1\right)\right| \geq \kappa$. If $0<r<\eta<\eta_{0}$ and $r^{-m}\left|B\left(y_{0}, r\right)\right|=\left(1-\frac{\delta_{0}}{2}\right) \omega_{m}$, then for every metric space $Z$, we have

$$
\inf _{r<s<\eta} s^{-1} d_{G H}\left(B\left(y_{0}, s\right), B\left(\left(\underline{0}, z^{*}\right), s\right)\right) \geq \eta,
$$

where $z^{*}$ is the vertex of the metric cone $C(Z),\left(\underline{0}, z^{*}\right) \in \mathbb{R}^{m-1} \times C(Z)$.

Proof. Otherwise, there exist a sequence of positive numbers $\eta_{i} \rightarrow 0$ and a sequence of Riemannian manifolds $\left(Y_{i}, y_{i}, h_{i}\right)$ with the given conditions violating the statements.

- $r_{i}^{-m}\left|B\left(y_{i}, r_{i}\right)\right|_{d \mu_{h_{i}}}=\left(1-\delta_{0}\right) \omega_{m}$ for some $0<r_{i}<\eta_{i}$.

- There exists $s_{i} \in\left(r_{i}, \eta_{i}\right)$ such that $s_{i}^{-1} d_{G H}\left(B\left(y_{i}, s_{i}\right), B\left(\left(\underline{0}, z_{i}^{*}\right), s_{i}\right)\right)<\eta_{i}$ for some $\mathbb{R}^{m-1} \times C\left(Z_{i}\right)$.

Let $\tilde{h}_{i}=s_{i}^{-2} h_{i}$. Denote the pointed-Gromov-Hausdorff limit of $\left(B_{\tilde{h}_{i}}\left(y_{i}, 1\right), y_{i}, \tilde{h}_{i}\right)$ by $(\hat{B}, \hat{y}, \hat{g})$. By limit process, there exists a metric space $\hat{Z}$ such that

$$
\hat{y}=\left(\underline{0}, \hat{z}^{*}\right) \in \mathbb{R}^{m-1} \times C(\hat{Z}), \hat{B}=B\left(\left(\underline{0}, \hat{z}^{*}\right), 1\right) .
$$

Clearly, every tangent space of $\hat{y}$ is $\mathbb{R}^{m-1} \times C(\hat{Z})$, which must be $\mathbb{R}^{m}$, due to Theorem 6.2 of [6]. Therefore, by the continuity of volume, we have

$$
\lim _{i \rightarrow \infty} s_{i}^{-m}\left|B_{h_{i}}\left(y_{i}, s_{i}\right)\right|_{d \mu_{h_{i}}}=\lim _{i \rightarrow \infty}\left|B_{\tilde{h}_{i}}\left(y_{i}, 1\right)\right|_{d \mu_{\tilde{h}_{i}}}=\omega_{m}
$$

which yields

$$
\left(1-\frac{1}{2} \delta_{0}\right) \omega_{m}=\lim _{i \rightarrow \infty} r_{i}^{-m}\left|B_{h_{i}}\left(y_{i}, r_{i}\right)\right|_{d \mu_{h_{i}}} \geq \lim _{i \rightarrow \infty} s_{i}^{-m}\left|B_{h_{i}}\left(y_{i}, s_{i}\right)\right|_{d \mu_{h_{i}}}=\omega_{m}
$$

by volume comparison. Contradiction!

Suppose $r_{V}(y)=1$. Let $y_{i} \rightarrow y$ as $\left(X_{i}, x_{i}, g_{i}\right)$ converges to $(\bar{X}, \bar{x}, \bar{g})$. Applying inequality (53) to the flow $\left\{\left(X_{i}, y_{i}, g_{i}(t)\right), 0 \leq t \leq 1\right\}$, we have $|R m|(y)=$ $\lim _{i \rightarrow \infty}|R m|_{g_{i}\left(\delta_{0}\right)}\left(y_{i}\right) \leq \delta_{0}^{-1}$. By a trivial rescaling argument, we see that

$$
|R m|(y) \min \left\{r_{V}^{2}(y), 1\right\} \leq \delta_{0}^{-1}
$$

for every $y \in \mathcal{R}$. Follow the route of [12] for the Einstein case, we can obtain some bounds of curvature integration on $\mathcal{R}$.

Proposition 5.3. For every $0<p<1$ and $\rho \geq 1$, we have a constant $C=$ $C(m, \kappa, p, \rho)$ such that

$$
\int_{B(\bar{x}, \rho) \cap \mathcal{R}}|R m|^{p} d \mu<C .
$$


Proof. Without loss of generality, we assume $\rho=1$. Fix $\eta<(1-p)$, we have

$$
\begin{gathered}
\delta_{0}^{p} \int_{B(\bar{x}, 1)}|R m|^{p} d \mu<\int_{B(\bar{x}, 1)} \min \left\{r_{V}^{-2 p}, 1\right\} d \mu \\
<C\left(1+\frac{1}{1-2^{2(p-1)+\eta}}\right)<C(m, \kappa, p),
\end{gathered}
$$

where we used (104) and (106).

Proposition 5.4. $\operatorname{dim}_{\mathcal{H}} \mathcal{S} \leq m-2$.

Proof. It follows from inequality (104) and the fact that $\operatorname{dim}_{\mathcal{H}} \mathcal{S}$ is always bounded by $m-k$ where $m-k$ is the largest integer where a singular tangent space can split off $\mathbb{R}^{m-k}$, which was proved in Theorem 4.7 of [6]. Alternatively, one can apply Theorem 6.1 of $[\underline{6}$ directly since our definition of regular-singular decomposition coincides with theirs a priori.

Combining all the discussions in this subsection, we finish the proof of Theorem[1.

5.2. Kähler case. Suppose $\left(M_{i}, x_{i}, g_{i}, J_{i}\right)$ is a sequence of almost Kähler Einstein manifolds. Let $(\bar{M}, \bar{x}, \bar{g})$ be the limit space of $\left(M_{i}, x_{i}, g_{i}\right), \bar{\lambda}$ be the limit of $\lambda_{i}$, and $\bar{M}=\mathcal{R} \cup \mathcal{S}$ be the regular-singular decomposition.

It is not hard to see that $\mathcal{R}$ has a complex structure $\bar{J}$ compatible with $\bar{g}$ and $\nabla_{\bar{g}} \bar{J}=0$. Actually, it suffices to prove the existence of such $\bar{J}$ locally. Fix $y \in \mathcal{R}$. Let $r_{0}=\frac{1}{2} r_{V}(y)$. Suppose $y_{i} \rightarrow y$ as $\left(M_{i}, x_{i}, g_{i}\right)$ converges to $(\bar{M}, \bar{x}, \bar{g})$. By the construction of $\bar{g}$, we know that $B_{\bar{g}}\left(y, r_{0}\right)$ is the smooth limit of $\left(B_{g_{i}\left(\delta_{0} r_{0}^{2}\right)}\left(y_{i}, r_{0}\right), g_{i}\left(\delta_{0} r_{0}^{2}\right)\right)$. Therefore, the complex structure $J_{i}$ on $B_{g_{i}\left(\delta_{0} r_{0}^{2}\right)}\left(y_{i}, \delta_{0} r_{0}^{2}\right)$ converges to the limit complex structure $\bar{J}$, which is compatible with $\bar{g}$ and $\nabla_{\bar{g}} \bar{J}=0$.

For the non-collapsed limit of Kähler manifolds with bounded Ricci curvature, it was known that every non-Euclidean tangent cone can split at most $2 n-4$ independent lines 5 The argument was based on an $\epsilon$-regularity theorem (cf Theorem 5.2 of [10]), which can be improved to obtain the following Lemma.

Lemma 5.2. There exists a constant $\xi_{0}=\xi_{0}(n, \kappa)$ with the following property.

Suppose $\left(N, y_{0}, h, J\right)$ is a complete Kähler manifold of complex dimension $n$, Ric $\geq-(n-1)$ on $N,\left|B\left(y_{0}, 1\right)\right| \geq \kappa$. Suppose for the scales $0<r<\eta<\xi_{0}$, we have

$$
\begin{aligned}
& \text { - } r^{-2 n}\left|B\left(y_{0}, r\right)\right|=\left(1-\frac{\delta_{0}}{2}\right) \omega_{2 n} . \\
& \text { - } \sup _{r<s<\eta} s^{2-2 n} \int_{B\left(y_{0}, 10 s\right)}|\operatorname{Ric}| d \mu<\eta .
\end{aligned}
$$

Then for every metric space $Z$, we have

$$
\inf _{r<s<\eta} s^{-1} d_{G H}\left(B\left(y_{0}, s\right), B\left(\left(\underline{0}, z^{*}\right), s\right)\right) \geq \eta,
$$

where $z^{*}$ is the vertex of the metric cone $C(Z),\left(\underline{0}, z^{*}\right) \in \mathbb{R}^{2 n-3} \times C(Z)$.

\footnotetext{
${ }^{5}$ This was proved by Cheeger in Theorem 6.1 of [10] and was also observed independently by the first author. As written by Cheeger on page 21 of [10, this result "requires no new ideas beyond those introduced in [8]."
} 
Proof. The proof follows the same route as that of Lemma 5.1

If the statement was wrong, there exist a sequence of scales $\left(r_{i}, \eta_{i}\right)$ with $\eta_{i} \rightarrow 0$ and a sequence of Kähler manifolds $\left(N_{i}, y_{i}, h_{i}, J_{i}\right)$ with the given conditions violating the statements:

- $r_{i}^{-2 n}\left|B\left(y_{i}, r_{i}\right)\right|_{d \mu_{h_{i}}}=\left(1-\frac{\delta_{0}}{2}\right) \omega_{2 n}$ for some $0<r_{i}<\eta_{i}$.

- $\sup _{r_{i}<s<\eta_{i}} s^{-2 n+2} \int_{B\left(y_{i}, 10 s\right)}|R i c|_{h_{i}} d \mu_{h_{i}}<\eta_{i}$.

- There exists $s_{i} \in\left(r_{i}, \eta_{i}\right)$ such that $s_{i}^{-1} d_{G H}\left(B\left(y_{i}, s_{i}\right), B\left(\left(\underline{0}, z_{i}^{*}\right), s_{i}\right)\right)<\eta_{i}$ for some $\mathbb{R}^{2 n-3} \times C\left(Z_{i}\right)$.

Let $\tilde{h}_{i}=s_{i}^{-2} h_{i}$. Denote the pointed-Gromov-Hausdorff limit of $\left(B_{\tilde{h}_{i}}\left(y_{i}, 1\right), y_{i}, \tilde{h}_{i}\right)$ by $(\hat{B}, \hat{y}, \hat{g})$. By limit process, there exists a metric space $\hat{Z}$ such that

$$
\hat{y}=\left(\underline{0}, \hat{z}^{*}\right) \in \mathbb{R}^{2 n-3} \times C(\hat{Z}), \hat{B}=B\left(\left(\underline{0}, \hat{z}^{*}\right), 1\right) .
$$

Like the proof of Lemma 5.1 in order to obtain a contradiction, it suffices to show that $\mathbb{R}^{2 n-3} \times C(\hat{Z})$ is isometric to $\mathbb{R}^{2 n}$. Actually, the Kähler condition implies that $\mathbb{R}^{2 n-3} \times C(\hat{Z})$ is either $\mathbb{R}^{2 n}$ or $\mathbb{R}^{2 n-2} \times C\left(S_{t}\right)$ for some circle with length $t \in(0,2 \pi)$. However, for metric $\tilde{h}_{i}$, we have

$$
\begin{aligned}
& \int_{B_{\tilde{h}_{i}\left(y_{i}, 10\right)}|R i c|_{\tilde{h}_{i}} d \mu_{\tilde{h}_{i}}=s_{i}^{-2 n+2}}|R i c|_{h_{i}} d \mu_{h_{i}} \leq \sup _{r_{i}<s<\eta_{i}} s^{-2 n+2} \int_{B_{h_{i}}\left(y_{i}, 10 s\right)}|R i c|_{h_{i}} d \mu_{h_{i}}<\eta_{i} \rightarrow 0 . \\
& \quad \int_{B_{h_{i}}\left(y_{i}, 10 s_{i}\right)}
\end{aligned}
$$

This is enough for us to choose good surfaces $D_{i}$, as slices of harmonic approximation maps, such that the integration of $|R i c|$ on $D_{i}$ is tending to zero (cf. Theorem 5.2 of [10]). In terms of Chern-Simons theory, the first Chern class character $\hat{c}_{1}$ is represented by $\frac{1}{2 \pi} \operatorname{Ric}(J \cdot, \cdot)$. The integration of $\hat{c}_{1}$ on $D_{i}$ then tends to zero. On the other hand, integration by parts implies that this integration is almost $1-\frac{t}{2 \pi}$. This forces that $t=2 \pi$. Actually, after we obtain the good slices with $\mid$ Ric|-integral small, all the other argument follows verbatim of that in Theorem 5.2 of [10]. Consequently, $\mathbb{R}^{2 n-3} \times C(\hat{Z})$ must be isometric to $\mathbb{R}^{2 n}$, and we can obtain the desired contradiction!

Fix the pair $(r, \eta)$ such that $0<r<\eta<\xi_{0}$. Let $y$ be an arbitrary point in $B(\bar{x}, 2) \subset \bar{M}, y_{i} \in M_{i}$ such that $y_{i} \rightarrow y$ as $\left(M_{i}, x_{i}, g_{i}\right)$ converges to $(\bar{M}, \bar{x}, \bar{g})$. Recall that $F_{i}=\int_{M_{i}}\left|R i c+\lambda_{i} g_{i}\right|_{g_{i}} d \mu_{g_{i}} \rightarrow 0$. For every $s \in(r, \eta)$, we have

$$
\begin{aligned}
& s^{2-2 n} \int_{B_{g_{i}}\left(y_{i}, 10 s\right)}|R i c|_{g_{i}} d \mu_{g_{i}} \\
& \quad \leq s^{2-2 n} \int_{B_{g_{i}}\left(y_{i}, 10 s\right)}\left\{\left|R i c+\lambda_{i} g_{i}\right|_{g_{i}}+\left|\lambda_{i}\right| \sqrt{n}\right\} d \mu_{g_{i}} \\
& \quad \leq r^{2-2 n} \int_{M_{i}}\left|R i c+\lambda_{i} g_{i}\right|_{g_{i}} d \mu_{g_{i}}+\left|\lambda_{i}\right| \sqrt{n}\left(s^{-2 n}\left|B_{g_{i}}\left(y_{i}, 10 s\right)\right|_{d \mu_{g_{i}}}\right) s^{2} \\
& \quad \leq r^{2-2 n} F_{i}+2 \sqrt{n} \cdot \omega_{2 n} \cdot 10^{2 n} \cdot \eta^{2} .
\end{aligned}
$$


It follows that

$$
\begin{aligned}
\sup _{r<s<\eta} s^{2-2 n} \int_{B_{g_{i}}\left(y_{i}, 10 s\right)}|R i c|_{g_{i}} d \mu_{g_{i}} & \leq r^{2-2 n} F_{i}+2 \sqrt{n} \cdot \omega_{2 n} \cdot 10^{2 n} \cdot \eta^{2} \\
& \leq 4 \sqrt{n} \cdot \omega_{2 n} \cdot 10^{2 n} \cdot \eta^{2}<\eta
\end{aligned}
$$

for large $i$, whenever $\eta$ is chosen very small. Therefore, Lemma 5.2 can be applied to obtain that $\mathcal{V}_{r} \subset \mathcal{S}_{\eta,(r, \eta)}^{2 n-4}$ on the limit space $\bar{M}$. Then we can apply Theorem 1.10 of [12] to obtain that

$$
\left|B(\bar{x}, 2) \cap \mathcal{V}_{r}\right| \leq C(n, \kappa, \eta) r^{4-\eta} .
$$

From here, we can deduce the following two propositions without difficulty.

Proposition 5.5. For every $0<p<2$ and $\rho \geq 1$, we have a constant $C=$ $C(m, \kappa, p, \rho)$ such that

$$
\int_{B(\bar{x}, \rho) \cap \mathcal{R}}|R m|^{p} d \mu<C
$$

Proposition 5.6. $\operatorname{dim}_{\mathcal{H}} \mathcal{S} \leq m-4$.

Combining all the discussion in this section, we finish the proof of Theorem 2 Moreover, Theorem 2 can be improved if we assume $\int_{M_{i}}|R m|_{g_{i}}^{p} d \mu_{g_{i}}<C$ uniformly for some $2 \leq p \leq \frac{m}{2}$, or we assume $n=p=2$. Instead of the dimension estimate of the singular set, one can actually have an effective bound of the volume of the $\epsilon$-neighborhood of the singular set in each unit ball. The proofs follow from the combination of the methods described in this section and that in 12. Since the proofs do not contain a new method and we do not know substantial applications of such results, we omit the details here.

\section{ExAmples}

In this section, we show two examples of almost Kähler Einstein sequences. The applications of the structure theorem (Theorem 2) are also discussed. Actually, both examples come to our attention spontaneously when we try to study the geometric properties of Kähler manifolds. It is for this study that we develop the whole paper.

6.1. Smooth minimal varieties of general type. A smooth projective variety $M$ is called of general type if the Kodaira dimension of $M$ is equal to the complex dimension of $M$, i.e.,

$$
\lim _{k \rightarrow \infty} \frac{\log \operatorname{dim} H^{0}\left(K_{M}^{k}\right)}{\log k}=n .
$$

It is called minimal if $K_{M}$ is numerically effective (nef), i.e., $K_{M} \cdot C \geq 0$ for every effective curve $C \subset M$. Suppose $M$ is a smooth minimal variety of general type; then it is easy to see that $M$ admits a Kähler Einstein metric if and only if $K_{M}$ is ample, by Yau's solution of Calabi conjecture. Since there are a lot of smooth minimal varieties whose canonical classes are not ample, we cannot expect to find a Kähler Einstein metric on each smooth minimal variety of general type. However, on each such variety, we can construct a sequence of almost Kähler Einstein metrics. 
Theorem 6.1. Suppose $M$ is a smooth minimal projective variety of general type, $J$ is the default complex structure. Then there is a point $x_{0} \in M$ and a sequence of metrics $g_{i}$ with the following properties:

- $\lim _{i \rightarrow \infty}\left[\chi_{i}\right]=-2 \pi c_{1}(M)$ where $\chi_{i}$ is the metric form compatible with both $g_{i}$ and $J$.

- $\left(M, x_{0}, g_{i}, J\right)$ is an almost Kähler Einstein sequence.

Proof. There exists a non-negative $(1,1)$-current $\chi$ with $[\chi]=-2 \pi c_{1}(M)$. Fix an arbitrary metric form $\omega$ on $M$. Then for every $\epsilon>0,[\chi+\epsilon \omega]$ is a positive class. By Yau's solution of Calabi conjecture (cf. 4] and [4]), we can find a metric form $\chi_{\epsilon}$ such that $\operatorname{Ric}\left(\chi_{\epsilon}\right)+\chi_{\epsilon}=\epsilon \omega$. Let $g_{\epsilon}$ be the metric tensor compatible with both $\chi_{\epsilon}$ and $J$. Clearly, we have

$$
\operatorname{Ric}\left(g_{\epsilon}\right)+g_{\epsilon} \geq 0 .
$$

Then we run the normalized Ricci flow

$$
\frac{\partial}{\partial t} g=-R i c-g
$$

from the initial metric $g_{\epsilon}$. Denote the metric form at time $t$ by $\chi_{\epsilon, t}$. Whenever $\chi_{\epsilon, t}$ is well defined, it satisfies

$$
\left[\chi_{\epsilon, t}\right]=e^{-t}\left[\chi_{\epsilon}\right]+\left(1-e^{-t}\right)[\chi]=[\chi]+\epsilon e^{-t}[\omega]>0 .
$$

Therefore, for every $\epsilon>0$, the normalized Ricci flow initiating from $g_{\epsilon}$ exists forever (cf. 41] and [40]). In view of (6), the condition $R+n \geq 0$ is preserved by the flow. Therefore, we have

$$
\begin{aligned}
\int_{0}^{1} & \int_{M}|R+n| \chi_{\epsilon, t}^{n} d t \\
& =\int_{0}^{1} \int_{M}(R+n) \chi_{\epsilon, t}^{n} d t \\
& =n \int_{0}^{1}\left(\int_{M}\left(\chi_{\epsilon, t}-\chi\right) \wedge \chi_{\epsilon, t}^{n-1}\right) d t \\
& =n \epsilon \int_{0}^{1} e^{-t}\left(\int_{M} \omega \wedge\left(\chi+\epsilon e^{-t} \omega\right)^{n-1}\right) d t \\
& <n \epsilon \int_{0}^{1} e^{-t}\left(\int_{M} \omega \wedge(\chi+\omega)^{n-1}\right) d t \\
& =n C \epsilon .
\end{aligned}
$$

At time $t=0$, we have $\operatorname{Ric}\left(\chi_{\epsilon}\right)+\chi_{\epsilon} \geq 0$, which implies

$$
\int_{M}\left|R i c+\chi_{\epsilon}\right| \chi_{\epsilon}^{n} \leq \int_{M} \sqrt{n}(R+n) \chi_{\epsilon}^{n}=\epsilon \cdot n^{\frac{3}{2}} \int_{M} \omega \wedge(\chi+\epsilon \omega)^{n-1}<C(\chi, \omega) n^{\frac{3}{2}} \epsilon .
$$

In view of the study of complex Monge-Ampere equation theory (cf. [40]), there exists an algebraically defined subvariety $\mathcal{B} \subset M$ such that $\chi_{\epsilon} \stackrel{C^{\infty}}{\longrightarrow} \hat{\chi}, g_{\epsilon} \stackrel{C^{\infty}}{\longrightarrow} \hat{g}$ on $M \backslash \mathcal{B}$, whenever $\epsilon \rightarrow 0$. Since $\hat{g}$ is a smooth metric on $M \backslash \mathcal{B}$, we can choose a small convex geodesic ball $B_{\hat{g}}\left(x_{0}, 2 \xi_{0}\right) \subset M \backslash \mathcal{B}$. Let $\epsilon_{i} \rightarrow 0, g_{i}=g_{\epsilon_{i}}$. Then we have

$$
\left|B_{g_{i}}\left(x_{0}, 1\right)\right|_{d \mu_{g_{i}}} \geq\left|B_{g_{i}}\left(x_{0}, 2 \xi_{0}\right)\right|_{d \mu_{g_{i}}}>\left|B_{\hat{g}}\left(x_{0}, \xi_{0}\right)\right| \triangleq \kappa
$$


for large $i$. By definition, (109), (112), (110), and (111) together imply that $\left(M, x_{0}, g_{i}, J\right)$ is an almost Kähler Einstein sequence.

In the proof of Theorem 6.1 when $\omega$ and $x_{0}$ are fixed, the almost Kähler Einstein sequence depends on the choice of the sequence $\left\{\epsilon_{i}\right\}_{i=1}^{\infty}$. It is natural to ask whether the limit space depends on the choice of the sequence $\left\{\epsilon_{i}\right\}_{i=1}^{\infty}$. In fact, the answer is no. In 39 , we proved that every limit space $(\bar{M}, \bar{x}, \bar{g})$ is the metric completion of $\left(M \backslash \mathcal{B}, x_{0}, \hat{g}\right)$, which is independent of the choice of $\left\{\epsilon_{i}\right\}_{i=1}^{\infty}$. Another interesting question is whether $\bar{M}$ has a variety structure. Generally, we do not know the answer although this is expected. However, when $(M, J)$ satisfies the Chern number equality $\left\{c_{1}^{2}(M)-\frac{2(n+1)}{n} c_{2}(M)\right\} \cdot c_{1}^{n-2}(M)=0$, then $\bar{M}$ does have a projective variety structure. Actually, in [39], we will use Theorem 2 to show that $\bar{M}$ is a global quotient of the complex hyperbolic space, and henceforth it is a variety.

6.2. Fano manifolds. A complex manifold $(M, J)$ is called a Fano manifold if $-K_{M}$ is ample. By the Kodaira embedding theorem, such a manifold must be projective and admits a Kähler structure. The existence of Kähler Einstein metrics on Fano manifolds is a hard problem (cf. 38 and references therein). In 36, the first author introduced the $\alpha$-invariant $\alpha(M)$ and proved that Kähler Einstein metrics exist whenever $\alpha(M)>\frac{n}{n+1}$. If we only assume $\alpha(M) \geq \frac{n}{n+1}$, then the situation becomes subtle. It is not clear whether $\alpha(M) \geq \frac{n}{n+1}$ implies the existence of Kähler Einstein metrics. On the other hand, the existence of Kähler Einstein metrics implies that Mabuchi's K-energy (cf. [29] for definition) is bounded from below. But there are examples (cf. [38, [15]) where the K-energy is bounded from below and Kähler Einstein metrics do not exist. In short, neither $\alpha(M) \geq \frac{n}{n+1}$ nor the K-energy bounded from below can guarantee the existence of Kähler Einstein metrics. However, either of them provides a sufficient condition for the existence of almost Kähler Einstein sequences.

Proposition 6.1. Suppose $(M, J)$ is a Fano manifold, $x_{0} \in M$. Then in the class $2 \pi c_{1}(M)$, there is a sequence of almost Kähler Einstein manifolds $\left(M, x_{0}, g_{i}, J\right)$ if one of the following conditions are satisfied:

- $\alpha(M) \geq \frac{n}{n+1}$.

- Mabuchi's K-energy is bounded from below in $2 \pi c_{1}(M)$.

Before we prove this proposition, let us recall an invariant. Suppose $(M, J)$ is a Fano manifold, and $\omega$ is a metric form in the class $2 \pi c_{1}(M)$. Since every other metric form in the same class can be written as $\omega_{\varphi}=\omega+\sqrt{-1} \partial \bar{\partial} \varphi$ for some smooth function $\varphi$ on $M$, it is clear that

$$
\sup \left\{t>0 \mid \operatorname{Ric}\left(\omega_{\varphi}\right) \geq t \omega_{\varphi} \text { for some } \varphi \in C^{\infty}(M)\right\}
$$

is independent of the choice of $\omega$. For brevity, we denote this invariant by $\mathcal{G}(M, J)$, or by $\mathcal{G}(M)$ when no ambiguity happens. Under this notation, we have the following theorem.

Theorem 6.2. Suppose $(M, J)$ is a Fano manifold with $\mathcal{G}(M)=1, x_{0} \in M$. Then there is a sequence of metrics $g_{i}$ with the following properties:

- $\left[\omega_{i}\right] \in 2 \pi c_{1}(M)$ where $\omega_{i}$ is the metric form compatible with both $g_{i}$ and $J$.

- $\left(M, x_{0}, g_{i}, J\right)$ is an almost Kähler Einstein sequence. 
Proof. Since $\mathcal{G}(M)=1$, for every $0<\alpha<1$, there is a metric form $\omega_{\alpha}$ with $\operatorname{Ric}\left(\omega_{\alpha}\right) \geq \alpha \omega_{\alpha}$. Let $g_{\alpha}$ be the metric tensor compatible with both $\omega_{\alpha}$ and $J$. Clearly, we have

$$
\operatorname{Ric}\left(g_{\alpha}\right) \geq \alpha g_{\alpha}
$$

Let $\alpha_{i} \rightarrow 1, \omega_{i}=\omega_{\alpha_{i}}$, and $g_{i}=g_{\alpha_{i}}$. Then we have

$$
\begin{aligned}
\int_{M}\left|R i c_{g_{i}}-g_{i}\right| \omega_{i}^{n} & \leq \int_{M}\left\{\left|R i c_{g_{i}}-\alpha_{i} g_{i}\right|+n\left(1-\alpha_{i}\right)\right\} \omega_{i}^{n} \\
& \leq \sqrt{n} \int_{M}\left\{R-n \alpha_{i}+n\left(1-\alpha_{i}\right)\right\} \omega_{i}^{n} \\
& =2 n^{\frac{3}{2}}\left(1-\alpha_{i}\right) \cdot(2 \pi)^{n} c_{1}^{n}(M) \rightarrow 0 .
\end{aligned}
$$

Initiating from $g_{i}$, we run the normalized Ricci flow

$$
\frac{\partial}{\partial t} g=-R i c+g
$$

which preserves the cohomology class $2 \pi c_{1}(M)$. Since $R-n \geq n\left(\alpha_{i}-1\right)$ at the initial time, it follows from (66) that

$$
(R-n)_{g_{i}(t)} \geq-n\left(1-\alpha_{i}\right) e^{t} \Rightarrow R_{g_{i}(t)} \geq n\left\{1-\left(1-\alpha_{i}\right) e^{t}\right\} .
$$

Consequently, we have

$$
\begin{aligned}
\int_{0}^{1} & \int_{M}|R-n|_{g_{i}(t)} \omega_{i}^{n}(t) d t \\
& =\int_{0}^{1} \int_{M}\left|R-n\left\{1-\left(1-\alpha_{i}\right) e^{t}\right\}-n\left(1-\alpha_{i}\right) e^{t}\right|_{g_{i}(t)} \omega_{i}^{n}(t) d t \\
& \leq \int_{0}^{1} \int_{M}\left\{R-n\left\{1-\left(1-\alpha_{i}\right) e^{t}\right\}+n\left(1-\alpha_{i}\right) e^{t}\right\} \omega_{i}^{n}(t) d t \\
& =2 n\left(1-\alpha_{i}\right) \cdot(2 \pi)^{n} c_{1}^{n}(M) \cdot \int_{0}^{1} e^{t} d t \\
& =2 n(e-1) \cdot(2 \pi)^{n} c_{1}^{n}(M) \cdot\left(1-\alpha_{i}\right) \rightarrow 0 .
\end{aligned}
$$

Since $\alpha_{i} \rightarrow 1$, we can assume $\alpha_{i}>\frac{2 n-1}{2 n}$. So the Bonnet-Myers theorem implies a diameter upper bound $\operatorname{diam}_{g_{i}} M<\sqrt{2 n} \pi$. By Bishop volume comparison, we have

$$
\frac{\left|B\left(x_{0}, 1\right)\right|_{d \mu_{g_{i}}}}{|M|_{d \mu_{g_{i}}}}=\frac{\left|B\left(x_{0}, 1\right)\right|_{d \mu_{g_{i}}}}{\left|B\left(x_{0}, \sqrt{2 n} \pi\right)\right|_{d \mu_{g_{i}}}} \geq C(n) \Rightarrow\left|B\left(x_{0}, 1\right)\right|_{d \mu_{g_{i}}} \geq C\left(n, c_{1}^{n}(M)\right) \triangleq \kappa,
$$

which is the non-collapsed condition. Therefore, by definition, (113), (117), (116), and (114) yield that $\left(M_{i}, x_{0}, g_{i}, J\right)$ form a sequence of almost Kähler Einstein manifolds.

Note that $\mathcal{G}(M)=1$ under either condition of Proposition 6.1 (cf. 35]). Therefore, Proposition 6.1 follows from Theorem 6.2

In both examples, Theorems 6.1 and 6.2 the complex structure is fixed. This is of course not needed in the setup of almost Kähler Einstein manifolds. Therefore, potentially, we should be able to construct almost Kähler Einstein sequences by deforming the complex structure and cohomology class simultaneously. It is then interesting to see whether the almost Kähler Einstein limit space is independent of 
the choice of parameter (of complex structures and metric forms) sequences. It is also fascinating to ask whether the limit space has a variety structure. These topics will be studied in the future.

\section{ACKNOWLEDGMENT}

The second author is very grateful to professor Xiuxiong Chen and professor Simon Donaldson for their constant support. He appreciates SCGP (Simons Center for Geometry and Physics) for offering him the wonderful working conditions. Part of this work was done when the second author was visiting BICMR (Beijing International Center of Mathematical Research) during the summer of 2011, and he would like to thank BICMR for its hospitality.

\section{REFERENCES}

[1] Michael T. Anderson, Ricci curvature bounds and Einstein metrics on compact manifolds, J. Amer. Math. Soc. 2 (1989), no. 3, 455-490, DOI 10.2307/1990939. MR999661 (90g:53052)

[2] Michael T. Anderson, Convergence and rigidity of manifolds under Ricci curvature bounds, Invent. Math. 102 (1990), no. 2, 429-445, DOI 10.1007/BF01233434. MR.1074481(92c:53024)

[3] Shigetoshi Bando, Atsushi Kasue, and Hiraku Nakajima, On a construction of coordinates at infinity on manifolds with fast curvature decay and maximal volume growth, Invent. Math. 97 (1989), no. 2, 313-349, DOI 10.1007/BF01389045. MR.1001844 (90c:53098)

[4] E. Calabi, The space of Kähler metrics, Proceedings of the International Congress of Mathematicians, Amsterdam, 1954, Vol. 2, Noordhoff, Groningen, 1954, pp. 206-207.

[5] Huai Dong Cao, Deformation of Kähler metrics to Kähler-Einstein metrics on compact Kähler manifolds, Invent. Math. 81 (1985), no. 2, 359-372, DOI 10.1007/BF01389058. MR:799272 (87d:58051)

[6] Jeff Cheeger and Tobias H. Colding, On the structure of spaces with Ricci curvature bounded below. I, J. Differential Geom. 46 (1997), no. 3, 406-480. MR1484888 (98k:53044)

[7] Jeff Cheeger and Tobias H. Colding, On the structure of spaces with Ricci curvature bounded below. III, J. Differential Geom. 54 (2000), no. 1, 37-74. MR.1815411 (2003a:53044)

[8] J. Cheeger, T. H. Colding, and G. Tian, On the singularities of spaces with bounded Ricci curvature, Geom. Funct. Anal. 12 (2002), no. 5, 873-914, DOI 10.1007/PL00012649. MR 1937830 (2003m:53053)

[9] Jeff Cheeger, A lower bound for the smallest eigenvalue of the Laplacian, Problems in analysis (Papers dedicated to Salomon Bochner, 1969), Princeton Univ. Press, Princeton, N.J., 1970, pp. 195-199. MR0402831(53 \#6645)

[10] J. Cheeger, Integral bounds on curvature elliptic estimates and rectifiability of singular sets, Geom. Funct. Anal. 13 (2003), no. 1, 20-72, DOI 10.1007/s000390300001. MR 1978491 (2004i:53041)

[11] Jeff Cheeger, Degeneration of Riemannian metrics under Ricci curvature bounds, Lezioni Fermiane. [Fermi Lectures], Scuola Normale Superiore, Pisa, 2001. MR2006642 (2004j:53049)

[12] Jeff Cheeger and Aaron Naber, Lower bounds on Ricci curvature and quantitative behavior of singular sets, Invent. Math. 191 (2013), no. 2, 321-339, DOI 10.1007/s00222-012-0394-3. MR.3010378

[13] Jeff Cheeger, Mikhail Gromov, and Michael Taylor, Finite propagation speed, kernel estimates for functions of the Laplace operator, and the geometry of complete Riemannian manifolds, J. Differential Geom. 17 (1982), no. 1, 15-53. MR658471 (84b:58109)

[14] Bennett Chow, Peng Lu, and Lei Ni, Hamilton's Ricci flow, Graduate Studies in Mathematics, vol. 77, American Mathematical Society, Providence, RI; Science Press, New York, 2006. MR2274812(2008a:53068)

[15] Xiuxiong Chen, The space of Kähler metrics, J. Differential Geom. 56 (2000), no. 2, 189-234. MR $1863016(2003 \mathrm{~b}: 32031)$

[16] Xiuxiong Chen, Claude Lebrun, and Brian Weber, On conformally Kähler, Einstein manifolds, J. Amer. Math. Soc. 21 (2008), no. 4, 1137-1168, DOI 10.1090/S0894-0347-08-00594-8. MR2425183(2010h:53054) 
[17] S. Y. Cheng and S. T. Yau, Differential equations on Riemannian manifolds and their geometric applications, Comm. Pure Appl. Math. 28 (1975), no. 3, 333-354. MR0385749(52 \#6608)

[18] Tobias H. Colding, Large manifolds with positive Ricci curvature, Invent. Math. 124 (1996), no. 1-3, 193-214, DOI 10.1007/s002220050050. MR1369415(96k:53068)

[19] Tobias H. Colding, Ricci curvature and volume convergence, Ann. of Math. (2) 145 (1997), no. 3, 477-501, DOI 10.2307/2951841. MR.1454700 (98d:53050)

[20] Tobias Holck Colding and Aaron Naber, Sharp Hölder continuity of tangent cones for spaces with a lower Ricci curvature bound and applications, Ann. of Math. (2) 176 (2012), no. 2, 1173-1229, DOI 10.4007/annals.2012.176.2.10. MR.2950772

[21] Lawrence C. Evans, Partial differential equations, 2nd ed., Graduate Studies in Mathematics, vol. 19, American Mathematical Society, Providence, RI, 2010. MR2597943 (2011c:35002)

[22] Mikhael Gromov, Structures métriques pour les variétés riemanniennes (French), Textes Mathématiques [Mathematical Texts], vol. 1, CEDIC, Paris, 1981. Edited by J. Lafontaine and P. Pansu. MR682063 (85e:53051)

[23] M. Gromov, Isoperimetric inequalities in Riemannian manifold, Asymptotic Theory of Finite Dimensional Normed Spaces, Textes Mathématiques [Mathematical Texts], vol. 1200, 1986.

[24] Leonard Gross, Logarithmic Sobolev inequalities and contractivity properties of semigroups, Dirichlet forms (Varenna, 1992), Lecture Notes in Math., vol. 1563, Springer, Berlin, 1993, pp. 54-88, DOI 10.1007/BFb0074091. MR.1292277 (95h:47061)

[25] D. Gilbarg and N. S. Trudinger, Elliptic partial differential equations of second order, Springer, Berlin, 1998.

[26] Richard S. Hamilton, Three-manifolds with positive Ricci curvature, J. Differential Geom. 17 (1982), no. 2, 255-306. MR664497 (84a:53050)

[27] Richard S. Hamilton, The formation of singularities in the Ricci flow, Surveys in differential geometry, Vol. II (Cambridge, MA, 1993), Int. Press, Cambridge, MA, 1995, pp. 7-136. MR 1375255 (97e:53075)

[28] P. Li, Lecture Notes on Geometric Analysis. available at http://math.uci.edu/ pli/lecture.pdf.

[29] Toshiki Mabuchi, K-energy maps integrating Futaki invariants, Tohoku Math. J. (2) 38 (1986), no. 4, 575-593, DOI 10.2748/tmj/1178228410. MR867064(88b:53060)

[30] G. Perelman, The entropy formula for the Ricci flow and its geometric applications, available at arXiv:math.DG/0211159.

[31] P. Petersen and G. Wei, Relative volume comparison with integral curvature bounds, Geom. Funct. Anal. 7 (1997), no. 6, 1031-1045, DOI 10.1007/s000390050036. MR.1487753 (99c:53023)

[32] Peter Petersen and Guofang Wei, Analysis and geometry on manifolds with integral Ricci curvature bounds. II, Trans. Amer. Math. Soc. 353 (2001), no. 2, 457-478, DOI 10.1090/S00029947-00-02621-0. MR:1709777 (2002c:53055)

[33] O. S. Rothaus, Logarithmic Sobolev inequalities and the spectrum of Schrödinger operators, J. Funct. Anal. 42 (1981), no. 1, 110-120, DOI 10.1016/0022-1236(81)90050-1. MR620582 (83f:58080b)

[34] Wan-Xiong Shi, Ricci deformation of the metric on complete noncompact Riemannian manifolds, J. Differential Geom. 30 (1989), no. 2, 303-394. MR1010165 (90f:53080)

[35] Gábor Székelyhidi, Greatest lower bounds on the Ricci curvature of Fano manifolds, Compos. Math. 147 (2011), no. 1, 319-331, DOI 10.1112/S0010437X10004938. MR2771134 (2011m:32037)

[36] Gang Tian, On Kähler-Einstein metrics on certain Kähler manifolds with $C_{1}(M)>0$, Invent. Math. 89 (1987), no. 2, 225-246, DOI 10.1007/BF01389077. MR894378(88e:53069)

[37] G. Tian, On Calabi's conjecture for complex surfaces with positive first Chern class, Invent. Math. 101 (1990), no. 1, 101-172, DOI 10.1007/BF01231499. MR1055713 (91d:32042)

[38] Gang Tian, Kähler-Einstein metrics with positive scalar curvature, Invent. Math. 130 (1997), no. 1, 1-37, DOI 10.1007/s002220050176. MR.1471884(99e:53065)

[39] G. Tian and Bing Wang, On the Chern number inequality of minimal varieties. preprint.

[40] Gang Tian and Zhou Zhang, On the Kähler-Ricci flow on projective manifolds of general type, Chinese Ann. Math. Ser. B 27 (2006), no. 2, 179-192, DOI 10.1007/s11401-005-0533-x. MR:2243679 (2007c:32029)

[41] Hajime Tsuji, Existence and degeneration of Kähler-Einstein metrics on minimal algebraic varieties of general type, Math. Ann. 281 (1988), no. 1, 123-133, DOI 10.1007/BF01449219. MR.944606(89e:53075) 
[42] Bing Wang, On the conditions to extend Ricci flow, Int. Math. Res. Not. IMRN 8 (2008), Art. ID rnn012, 30, DOI 10.1093/imrn/rnn012. MR2428146 (2009k:53176)

[43] Bing Wang, On the conditions to extend Ricci flow(II), Int. Math. Res. Not. IMRN 14 (2012), 3192-3223, DOI 10.1093/imrn/rnr141. MR2946223

[44] Shing Tung Yau, On the Ricci curvature of a compact Kähler manifold and the complex Monge-Ampère equation. I, Comm. Pure Appl. Math. 31 (1978), no. 3, 339-411, DOI 10.1002/cpa.3160310304. MR480350(81d:53045)

Department of Mathematics, Princeton University, Princeton, New Jersey 08544; Beijing International Center for Mathematical Research, School of Mathematical Sciences, Peking University, Beijing 100871, People's Republic of China

E-mail address: tian@math.princeton.edu

Department of Mathematics, University of Wisconsin-Madison, Madison, Wisconsin 53706

E-mail address: bwang@math.wisc.edu 